ARTICLE

Received 7 Oct 2013 | Accepted 12 Mar 2014 | Published 9 Apr 2014 DOl: 10.1038/ncomms4639

\title{
Olfactory projectome in the zebrafish forebrain revealed by genetic single-neuron labelling
}

Nobuhiko Miyasaka ${ }^{1}$, Ignacio Arganda-Carreras ${ }^{2} \uparrow$, Noriko Wakisaka ${ }^{1}$, Miwa Masuda ${ }^{1}$, Uygar Sümbül$^{2}$, H. Sebastian Seung ${ }^{2, \dagger} \&$ Yoshihiro Yoshihara ${ }^{1}$

Chemotopic odour representations in the olfactory bulb are transferred to multiple forebrain areas and translated into appropriate output responses. However, a comprehensive projection map of bulbar output neurons at single-axon resolution is lacking in vertebrates. Here we unravel a projectome of the zebrafish olfactory bulb through genetic single-neuron tracing and image registration. We show that five major target regions receive distinct modes of projections from olfactory bulb glomeruli. The central portion of posterior telencephalon receives non-selective, interspersed inputs from all glomeruli, whereas the ventral telencephalon is diffusely innervated by axons from particular glomerular clusters. The right habenula and posterior tuberculum (diencephalic nuclei) receive convergent inputs from restricted and all glomerular clusters, respectively. The bulbar recurrent projections are coarsely topographic. Thus, the primary chemotopic organization is transformed into distinct sensory representations in higher olfactory centres. These findings provide a framework to understand general principles as well as species-specific features in decoding of odour information.

\footnotetext{
${ }^{1}$ RIKEN Brain Science Institute, 2-1 Hirosawa, Wako, Saitama 351-0198, Japan. ${ }^{2}$ Department of Brain and Cognitive Sciences, Massachusetts Institute of Technology, 43 Vassar Street, Cambridge, Massachusetts 02139, USA. † Present addresses: INRA, UMR1318, Institut Jean-Pierre Bourgin, 78026 Versailles, France (I.A.-C.); Princeton Neuroscience Institute, Princeton University, Princeton, New Jersey 08544, USA (H.S.S). Correspondence and requests for materials should be addressed to N.M. (email: miyasaka@brain.riken.jp) or to Y.Y. (email: yoshihara@brain.riken.jp).
} 
A nimals use the sense of smell to monitor chemical cues in their environment, which provide vital information for food searching, predator avoidance, mate choice and social interactions. The odour information is initially represented as a discrete pattern of neural activities across a glomerular array on the olfactory bulb (OB), which results from axonal convergence of olfactory sensory neurons (OSNs) expressing the same olfactory receptors ${ }^{1}$. Glomeruli responsive to structurally related odorants are often clustered within defined regions of the $\mathrm{OB}^{2-5}$, establishing a chemotopic map of molecular features, so-called odour map. The odour map in the $\mathrm{OB}$ is transmitted by output neurons, mitral cells, to higher olfactory centres and eventually translated to elicit appropriate behavioural and physiological responses. Recent anatomical studies in mice showed that axons from identified glomeruli project diffusely throughout the piriform cortex ${ }^{6-9}$, the largest target area of the $\mathrm{OB}$, and that piriform neurons receive convergent inputs from multiple mitral cells distributed throughout the $\mathrm{OB}^{10}$. These findings are consistent with an optical imaging study that found no apparent spatial organization of odour-evoked activity patterns in the piriform cortex ${ }^{11}$. In contrast, the anterior olfactory nucleus and the cortical amygdala receive topographic and biased projections from the $\mathrm{OB}$, respectively 9,10 . Thus, a conceptual organization has been proposed in which the secondary olfactory pathway bifurcates to transform odour information into stereotyped and random representations, features suited for directing innate and learned behaviours, respectively ${ }^{12,13}$. However, it is not entirely clear how projections of individual output neurons to multiple brain areas are organized, because each of these studies in mice analysed only a small fraction of mitral cells and/or a restricted subset of its target areas.

Understanding the whole picture of olfactory processing and perception in vertebrates requires comprehensive wiring diagrams from the $\mathrm{OB}$ to higher brain centres and beyond. One of the most informative approaches to this goal is the sparse labelling method that involves visualization of a single neuron per brain and the integration of information across many brain samples; this method has been successfully employed in the Drosophila olfactory system ${ }^{14-17}$. Among vertebrates, a promising model organism for connectivity mapping is the zebrafish because of its amenability to various genetic techniques, small brain and transparency at early larval stages suitable for high-resolution imaging ${ }^{18,19}$. We previously developed a genetic method that visualizes a subpopulation of $\mathrm{OB}$ output neurons at single-cell resolution and revealed patterns of axon projections originating mainly from a particular glomerular cluster to higher brain centres in zebrafish larvae ${ }^{20}$. Here, we further expand the analysis into output neurons innervating most classes of glomerular clusters and present three-dimensional (3D) projection maps by employing a state-of-the-art image registration method. Our data describe a comprehensive axon projection map of the secondary olfactory pathway, permitting us to make predictions about information transfer and to hypothesize on evolutionarily conserved principles as well as species-specific features of how odour information is translated into various physiological and behavioural responses.

\section{Results}

Two genetically distinct populations of $O B$ output neurons. Genetic tracing of the secondary olfactory pathway requires gene promoters that target $\mathrm{OB}$ output neurons. We previously reported lhx $2 a$ gene promoter that allows for visualizing a subpopulation of OB output neurons mainly innervating one glomerular cluster, mdG (formerly called mG) ${ }^{20}$ (Fig. 1a). Here we analysed zebrafish tbx21 gene promoter, because its orthologous murine gene is exclusively expressed in $\mathrm{OB}$ output neurons ${ }^{21}$ and the promoter successfully visualized the secondary olfactory pathway in transgenic mice ${ }^{22}$. We first established a transgenic zebrafish line in which DsRed and GFP are expressed under the control of $t b \times 21$ and $l h x 2 a$ promoters, respectively. DsRed expression driven by $t b \times 21$ promoter was observed preferentially in the ventromedial $\mathrm{OB}$ in addition to a small cluster of neurons in the anterodorsolateral telencephalon at 7 days postfertilization (d.p.f.), whereas $l h x 2 a: G F P$-expressing neurons were mainly localized in the mediodorsal $\mathrm{OB}$ as previously described ${ }^{20}$ (Fig. 1b-e). We never encountered overlapping signals of DsRed and GFP, indicating that $t b \times 21$ and $l h \times 2 a$ promoters drive transgene expression in distinct populations of $\mathrm{OB}$ neurons in a mutually exclusive manner (hereby referred to as $t b x 21^{t g+}$ and lh $x 2 a^{\text {tg }}+\mathrm{OB}$ neurons, respectively).

We next examined axon projection patterns of these two subpopulations of $\mathrm{OB}$ neurons by expressing membrane-tethered fluorescent proteins, tdTomato-CAAX and gap-YFP, in $t b \times 21^{t g+}$ and $l h \times 2 a^{t g+}$ neurons, respectively (Fig. If $-\mathrm{m}$ ). $t b \times 21^{t g}+\mathrm{OB}$ neurons extended axons outside the OB (Fig. 1g,k), indicating a characteristic of output neurons. $t b \times 21^{t g}+$ neurons elaborated axonal branches extensively in the telencephalon (Fig. 1g,h), whereas $\ln \times 2 a^{\operatorname{tg}+}$ neurons arborized fewer branches at a lateral region in the vicinity of anterior commissure (Fig. 1f,h). $\operatorname{lh} \times 2 a^{\operatorname{tg}+}$ neurons bilaterally formed tight fascicles that projected from the telencephalon towards the dorsal diencephalon, elaborating a terminal-like tuft in the right habenula $(\mathrm{rHb})$, but not in the left habenula $(\mathrm{lHb})^{20}$ (Fig. 1f,h). In contrast, $t b \times 21^{t g}+$ neurons showed only a few axons reaching the rHb (Fig. 1g,h). When viewed from the ventral side, we observed a considerable number of $t b \times 21^{t g}+$ axons running longitudinally through bilaterally parallel tracts and reaching the posterior tuberculum (PT), a ventral diencephalic nucleus close to the hypothalamus (Fig. 1k,l). $l h \times 2 a^{t g+}$ neurons showed a few axons projecting to the PT with the trajectory similar to that of $t b \times 21^{t g+}$ axons (Fig. 1j,ll). Thus, tbx21tg+ and $l h \times 2 a^{t g}+\mathrm{OB}$ output neurons exhibited conspicuous differences of axon projection patterns in higher brain centres (Supplementary Movies 1 and 2).

Single-neuron tracing of secondary olfactory projections. To analyse glomerular innervation and axonal trajectory of individual OB output neurons in more detail, we performed genetic mosaic labelling and whole-mount immunohistochemistry with anti-GFP and anti-SV2 antibodies, which reveal detailed neuronal morphology and overall brain architecture, respectively. By screening 2,632 larvae in total, we identified 113 larvae $(4.3 \%)$ that harboured single-labelled $\mathrm{OB}$ output neurons and obtained images of these neurons at high resolution with confocal microscopy.

Glomeruli in the larval $\mathrm{OB}$ at 7 d.p.f. can be divided topographically and structurally into eight clusters ${ }^{23}$ (mdG, dlG, dG, maG, vaG, vmG, vpG and lG; Fig. 1a), whose regional layout is essentially maintained into adulthood ${ }^{24,25}$. Individual output neurons exhibited a single dendritic tuft that occupied a single glomerulus or a confined area within a defined glomerular cluster (Fig. 2b). These included $22 \operatorname{lh} \times 2 a^{t g}+$ neurons, all of which innervated $\mathrm{mdG}$, and $91 \mathrm{tb} \times 21^{t g+}$ neurons, which innervated all glomerular clusters other than dlG. The vast majority of output neurons ( $16 \operatorname{lh} \times 2 a^{\operatorname{tg}+}$ and $\left.84 t b \times 21^{\operatorname{tg}+}\right)$ sent axons outside the $\mathrm{OB}$ through the presumptive medial olfactory tract, whereas the remaining neurons (6 lh $\times 2 a^{t g}+$ and 7 $t b \times 21^{\operatorname{tg}+}$ ) extended axons through the presumptive lateral olfactory tract or an intermediate zone. All output neurons elaborated axon branches in both hemispheres of the 
a

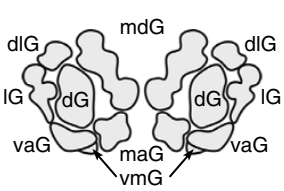

Frontal view

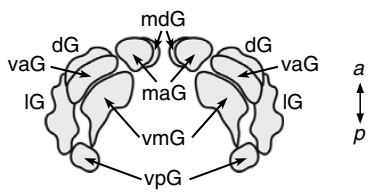

Ventral view
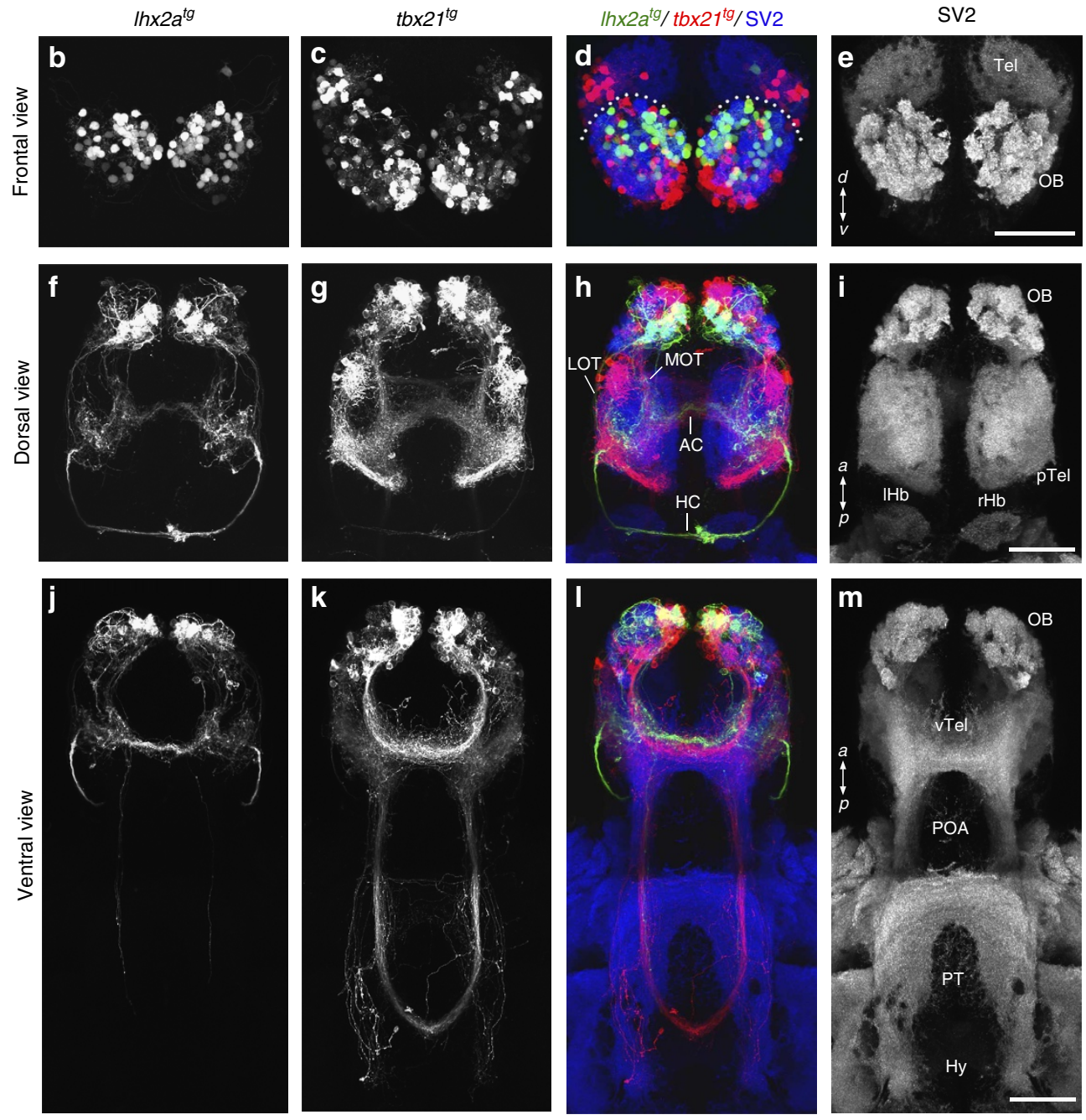

Figure 1 | Two distinct subpopulations of OB output neurons revealed in transgenic zebrafish. (a) Schematic drawings of eight glomerular clusters in the OB of 7-d.p.f. larvae viewed from the frontal and ventral sides. $d$, dorsal; $v$, ventral; $a$, anterior; $p$, posterior. (b-e) Confocal Z-stack images of OB output neurons visualized in a transgenic larva carrying Ihx2a:GFP, tbx21:Gal4 and UAS:DsRed transgenes. The whole-mount brain is stained with anti-GFP (b; green in d), anti-RFP ( $\mathbf{c}$; red in $\mathbf{d}$ ) and anti-SV2 (e; blue in $\mathbf{d}$ ) antibodies and is shown in the frontal view. Dotted lines in $\mathbf{d}$ indicate the boundary between the OB and the Tel. Scale bar, $50 \mu \mathrm{m}$. (f-m) Confocal Z-stack images of OB output neurons visualized in a transgenic larva carrying Ihx2a:gap-YFP, tbx21:Gal4 and UAS:tdTomato-CAAX transgenes. The whole-mount brain is stained with anti-GFP ( $\mathbf{f}$ and $\mathbf{j}$; green in $\mathbf{h}$ and $\mathbf{I}$ ), anti-RFP ( $\mathbf{g}$ and $\mathbf{k}$; red in $\mathbf{h}$ and $\mathbf{I}$ ) and anti-SV2 (i and $\mathbf{m}$; blue in $\mathbf{h}$ and $\mathbf{I}$ ) antibodies and is shown in the dorsal (f-i) and ventral (j-m) views. MOT, medial olfactory tract; LOT, lateral olfactory tract; $\mathrm{AC}$, anterior commissure; $\mathrm{HC}$, habenular commissure; $\mathrm{IHb}$, left habenula; $\mathrm{rHb}$, right habenula; POA, preoptic area; $\mathrm{Hy}$, hypothalamus. Scale bar, $50 \mu \mathrm{m}$.

telencephalon, which were interconnected by the axon crossing the midline through the anterior commissure and/or occasionally through the habenular commissure (Fig. 2b). The zebrafish habenulae display a prominent left-right asymmetry of neuropil structure ${ }^{26}$ with an expanded round neuropil area on the left and a medially elongated area on the right, which can be clearly defined by SV2 staining ${ }^{20}$ (Fig. 1i; Supplementary Fig. 1d-f). Of 113 larvae, 23 (20\%) showed reversed laterality of the habenulae; the incidence of spontaneous left-right reversal of the habenulae in our facility appeared to be higher than those in previous reports ${ }^{27,28}$ for unknown reason.
The $\mathrm{OB}$ output neurons projected axons to five major target regions: the posterior telencephalon ( $\mathrm{pTel}$ ), ventral telencephalon (vTel), rHb, PT and $\mathrm{OB}$ itself. The individual target regions received inputs from $\mathrm{OB}$ glomeruli (and glomerular clusters) in highly unique manners as described in detail below.

Projections to the posterior telencephalon. For the comparison of spatial patterns of axon projections in the telencephalon from different output neuron classes, it is essential to bring singleneuron images of different specimens into a common coordinate 
a

mdG

dG
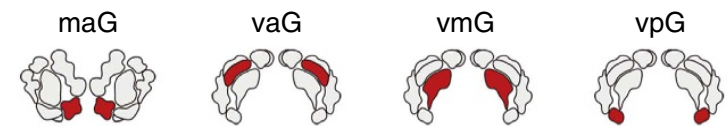

IG

b
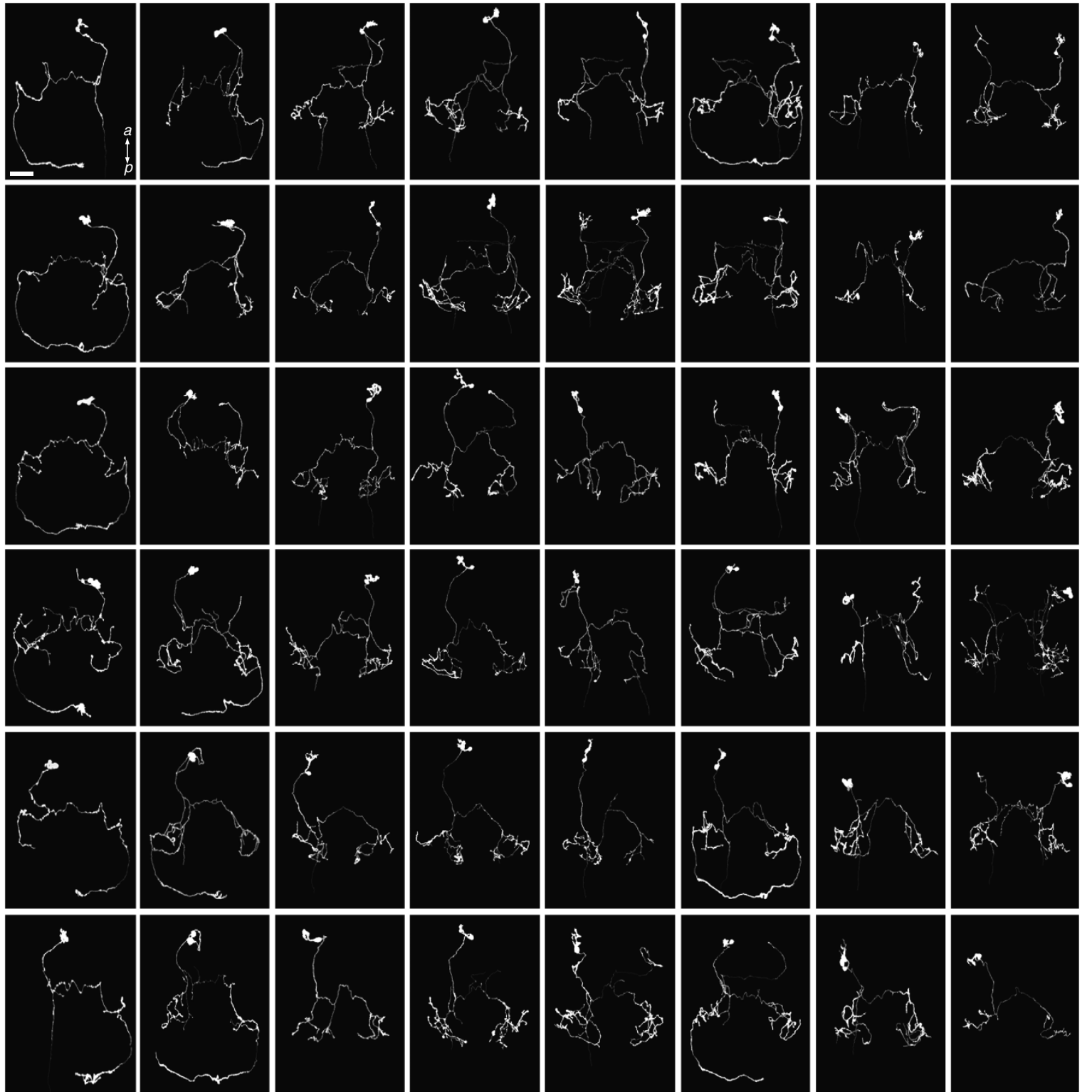

C
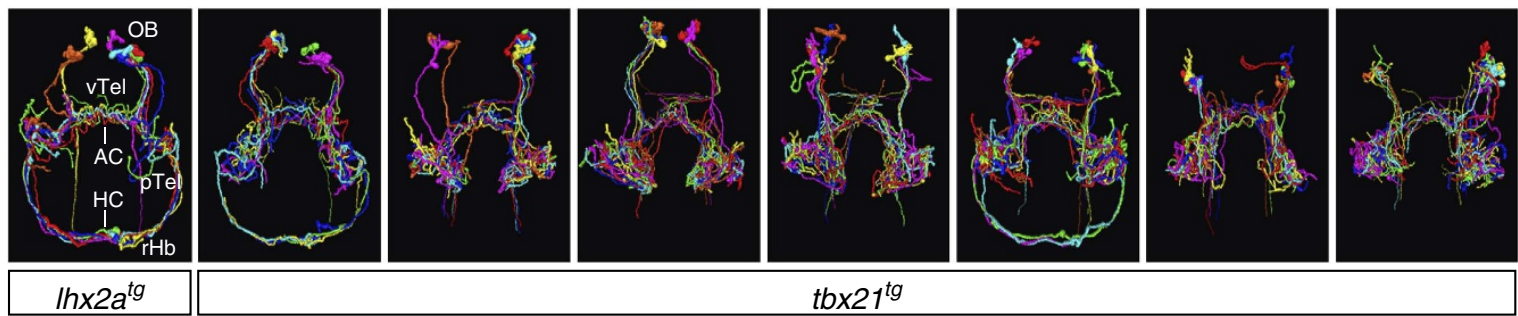

Figure 2 | Projection patterns of individual OB output neurons registered onto a common coordinate system. (a) Schematic drawings of glomerular clusters. (b) Images of six output neurons for each class (seven glomerular clusters; two transgenes for mdG; eight classes in total), after registration against the reference brain and subtraction of background stainings, are shown in the dorsal view with the anterior to the top. Scale bar, $30 \mu \mathrm{m}$.

(c) Three-dimensional renderings of registered OB output neurons. Six ( $m d G-t b \times 27^{t g}{ }^{+}$) or seven (other classes) neurons are simultaneously visualized in the dorsal view. Different colours represent different output neurons. Six of seven neurons used are shown in $\mathbf{b}$. 
a
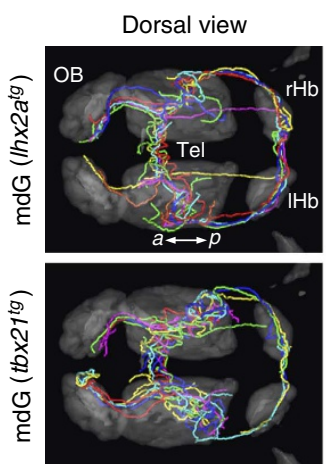

잉
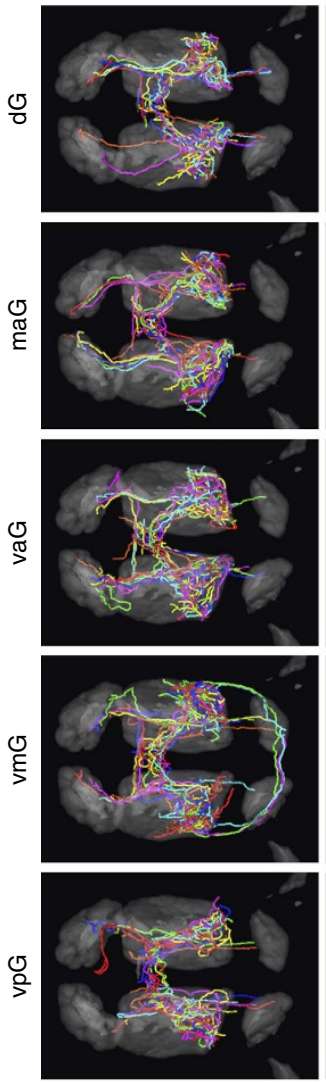

$\underline{0}$

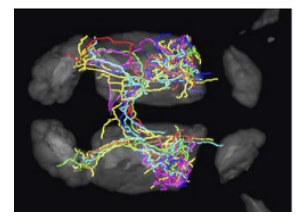

Lateral view
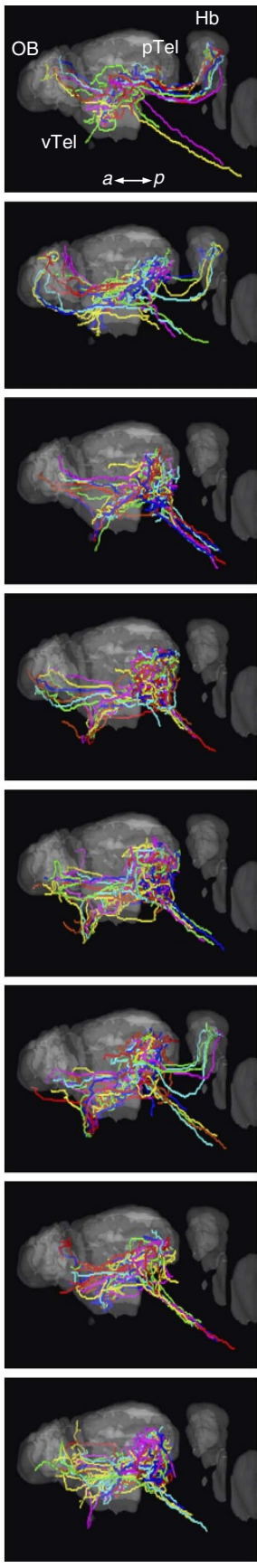

b
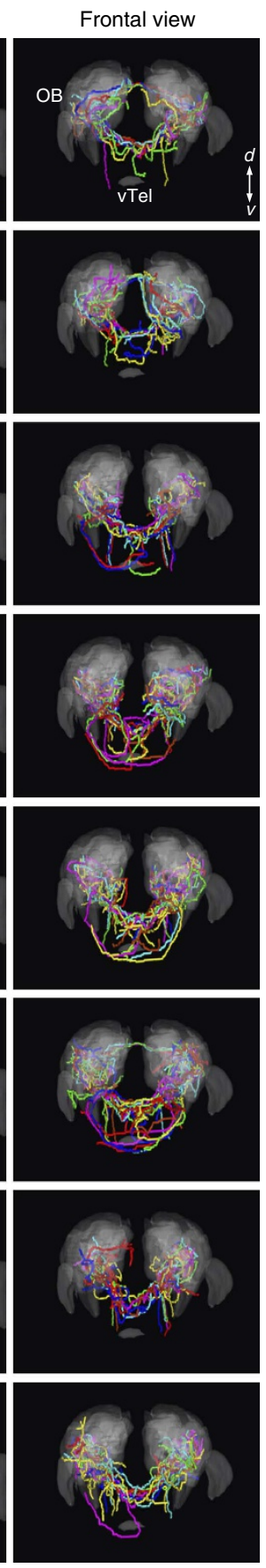

c vTel

pTel
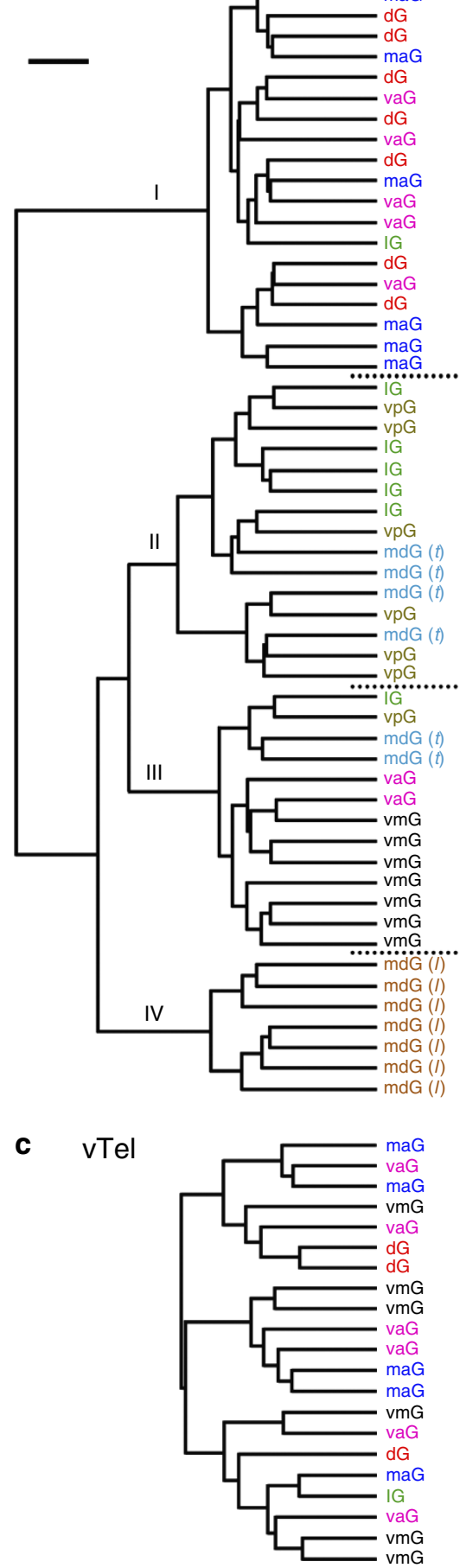

Figure 3 | Axon projection patterns for each class of OB output neurons. (a) Axon tracings of six (mdG-tbx $21^{\text {tg }}+$ ) or seven (other classes) output neurons are simultaneously visualized onto the SV2-stained reference brain (grey) in the dorsal, lateral and frontal views. Different colours represent different output neurons. (b) A dendrogram categorizes 55 output neurons into four large groups (I-IV) according to their similarity of axon projection patterns in the pTel. The linkage distances were calculated using Ward's method. The horizontal bar indicates 0.5 units in Ward's linkage distance.

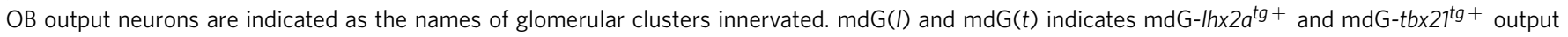
neurons, respectively. (c) OB output neurons that display projections to the vTel were selected (21 of 55 neurons in b) and cluster analysis was performed on these neurons with axon tracings within the vTel.

system. We therefore created a reference brain from 21 samples stained with SV2 and aligned single neurons onto the reference space using an intensity-based 3D image registration method ${ }^{29}$ (Supplementary Figs 2 and 3). The aligned neurons were traced in three dimensions and then background noises were subtracted from the images (Fig. 2b). These procedures allow us to simultaneously visualize arbitrary combinations of multiple output neurons in relation to glomerular classes.

Two major target areas in the telencephalon are the pTel and vTel, which would later give rise to the posterior zone of the 
dorsal telencephalic area (Dp) and the ventral nucleus of the ventral telencephalic area, respectively. All single-labelled output neurons projected axons to the pTel bilaterally with distributive and complex branching patterns (Figs 2 and 3a). On gross visual inspection, projections from different glomerular clusters appeared to be highly overlapping but to display different spatial patterns of axonal arborization. To quantitatively assess the spatial organization of axonal trajectories in the pTel, we performed cluster analysis on 55 output neurons innervating seven different glomerular clusters (Fig. $3 b$ ). The axonal tracings within the pTel for individual output neurons were convolved with a Gaussian function to represent projection areas and subjected to cluster analysis using Ward's method ${ }^{30}$. The resulting dendrogram categorized 55 neurons into four large groups; neurons innervating the same glomerular cluster did not always fall into the same group. The first group consisted mostly of three classes of output neurons, whose glomerular clusters (vaG, $\mathrm{maG}$ and $\mathrm{dG}$ ) are innervated by OSNs that express G-protein $\alpha$ subunit $G_{\text {olf }}$, olfactory marker protein and presumably OR-type olfactory receptors (presumptive ciliated OSNs) ${ }^{25,31,32}$. Each neuronal pair with highest similarity in this group often belonged to different glomerular clusters. The second group was composed of lG-, vpGand $\mathrm{mdG}\left(t b \times 21^{t g+}\right)$-neurons, which receive inputs from $\mathrm{G}_{\mathrm{olf}^{-}}$ negative OSNs presumably expressing either V1R- or V2R-type olfactory receptors (presumptive microvillous and crypt OSNs) ${ }^{25,31-35}$. The major population of the third group was vmG-neurons that mostly receive inputs from $G_{\text {olf-positive }}$ $\mathrm{OSNs}^{25}$. The fourth group consisted of a single class of output neurons, mdG-lhx $2 a^{\text {tg }}+$ neurons.

To directly visualize the spatial organization of axon projections in the pTel, we selected four different combinations of glomerular classes on the basis of cluster analysis and created overlaid $3 \mathrm{D}$ reconstructions of axonal tracings (Fig. 4). We first compared vaG-, maG- and dG-neurons, which mostly fall into the first group (Fig. 3b). Projections from these neuron classes largely overlapped and occupied a defined area in the pTel (Fig. 4a; Supplementary Movie 3). Individual axons, regardless of glomerular classes, were highly intermingled with each other within the defined area. We next examined two combinations (Fig. 4b,c), in which different glomerular classes of output neurons virtually fall into distinct groups on cluster analysis (Fig. 3b). Three ventral glomerular classes (vaG, vmG and vpG) of output neurons displayed overlapping projections especially within the central portion of their whole projection area. Peripheral portions, however, received biased inputs from specific glomerular clusters: medioposterior, laterodorsal and mediodorsal portions received axons predominantly from vaG (blue), vmG (red) and vpG (green), respectively (Fig. 4b; Supplementary Movie 4). Projections of maG- and lG-neurons overlapped, but appeared to coarsely preserve the medial-lateral topography from the $\mathrm{OB}$ to the pTel: medioposterior and lateroposterior portions received biased inputs from maG (red) and $\mathrm{lG}$ (green), respectively (Fig. 4c; Supplementary Movie 5). Lastly, we compared projections from $l h \times 2 a^{t g}+$ and $t b \times 21^{t g}+$ output neurons both innervating mdG (Fig. 4d; Supplementary Movie 6). $\operatorname{lh} \times 2 a^{t g}+$ and $t b \times 21^{t g}+$ neurons tended to innervate different sets of glomeruli within mdG (Supplementary Fig. 4), and their projections segregated along anterior-posterior axis in the pTel. Taken together, these findings demonstrate that the central portion of pTel receives non-selective and diffuse inputs from all the glomerular clusters, whereas the peripheral portions receive biased inputs from specific clusters.

Projections to the ventral telencephalon. Projections to the vTel were best visualized in frontal views of $3 \mathrm{D}$ reconstructions
(Fig. 3a, right column). A considerable population of output neurons innervating dG (36\%), maG (62\%), vaG (82\%) and vmG (55\%) displayed projections to the vTel; axon branches emerged from the anterior commissure or nearby telencephalic regions and reached the surface of the vTel, occasionally crossing the midline. These four glomerular clusters are all innervated by $\mathrm{G}_{\text {olf }}{ }^{-}$ positive OSNs. Projections to the vTel were observed for a smaller proportion of output neurons that receive inputs from $\mathrm{G}_{\mathrm{olf}^{-}}$ negative OSNs (mdG, $5 \%$ for $\operatorname{lh} \times 2 a^{\operatorname{tg}+}$ and $0 \%$ for $t b \times 21^{\operatorname{tg}+}$; $\mathrm{vpG}, 0 \% ; \mathrm{lG}, 18 \%)$. To assess the spatial organization of axonal trajectories in the vTel, we selected 21 neurons that display projections to the vTel from above-mentioned 55 neurons, and performed cluster analysis with axon tracings within the vTel. The resultant dendrogram exhibited no apparent neuronal group relating to specific glomerular classes (Fig. 3c), suggesting that there is no spatial segregation of projections in the vTel. Thus, the vTel receives diffuse inputs from a biased population of glomerular clusters.

Projections to the habenula. In addition to targeting the telencephalon, the majority of output neurons (87\%) exhibited projections to the diencephalon, each of which send axons to either the $\mathrm{Hb}(18 \%)$ or the PT $(52 \%)$ or both $(17 \%)$. The projections to the $\mathrm{Hb}$ were identified for restricted glomerular classes of output neurons: $87 \%$ of mdG-neurons (20 of $22 \operatorname{lh} \times 2 a^{t g+} ; 7$ of 9 tbx $\left.21^{t g+}\right), 50 \%$ of vmG-neurons (11 of 22 ) and $7 \%$ of dG-neurons (1 of 15). The axons reaching the dorsal diencephalon elaborated terminal-like tufts on a medial portion of the neuropil domain in the $\mathrm{rHb}$, but not in the $1 \mathrm{Hb}$ (Fig. 2b; Supplementary Fig. 1a-f). When larvae showed reversed laterality of the habenulae ( 8 of 39 larvae harbouring $\mathrm{Hb}$-projecting neurons), the axonal tufts were seen on the corresponding position in the $\mathrm{lHb}$ (Supplementary Fig. $1 \mathrm{~g}-\mathrm{l})$. These observations are in agreement with a recent report showing that directionality of habenular afferents from the $\mathrm{OB}$ depends on the laterality of habenulae ${ }^{36}$. Thus, the $\mathrm{rHb}$ (or the $\mathrm{lHb}$ of left-right reversed habenulae) receives convergent and highly biased inputs from select glomerular clusters.

Projections to the posterior tuberculum. The PT is a hypothalamus-related nucleus, containing populations of dopaminergic neurons that project to distant brain regions 37,38 . A large proportion of $t b \times 21^{t g}+$ neurons from every glomerular cluster $(63-100 \%)$ and a smaller proportion (33\%) of $l h \times 2 a^{t g}+$ neurons from $\mathrm{mdG}$ were observed to send a long axon to the PT (Fig. 5ae). These descending axons emanated from ventroposterior part of axonal arbors in the pTel (Fig. 3a, middle column), extended through the longitudinal tracts, and entered into the PT, often crossing the midline (Fig. 5a-e). These axons from the OB closely associated with tyrosine hydroxylase $(\mathrm{TH})$-positive processes elaborated by dopaminergic neurons in the PT (Fig. 5f-j). To locate presynaptic sites of $\mathrm{OB}$ output neurons in the PT, we crossed tbx21:Gal4 driver line with a UAS reporter line in which simultaneous expression of Synaptophysin-GFP fusion protein (Syp-GFP) and tdTomato-CAAX can be driven (Supplementary Fig. 5). Syp-GFP puncta were observed along the path of axons from the OB in the PT (Fig. 5k-o). Some of the puncta were located in close proximity to the TH-positive processes of dopaminergic neurons (Fig. 5p-t), suggesting possible synaptic connections between these neuronal populations.

Projections to the ipsi- and contralateral OBs. A proportion of $\mathrm{OB}$ output neurons sent axonal branches back into the OB. These processes emanated along or near the anterior commissure, occasionally run through the vTel, and eventually re-entered the $\mathrm{OB}$ of ipsilateral and/or contralateral sides relative to the soma 
a

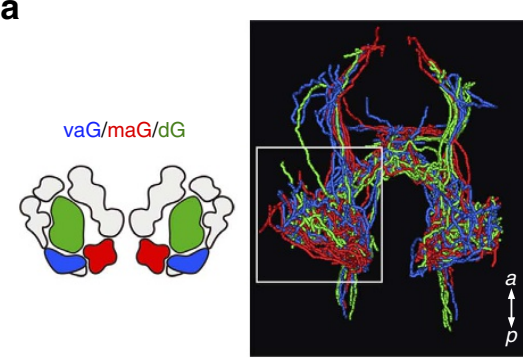

Dorsal view

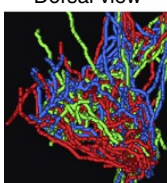

Lateral view
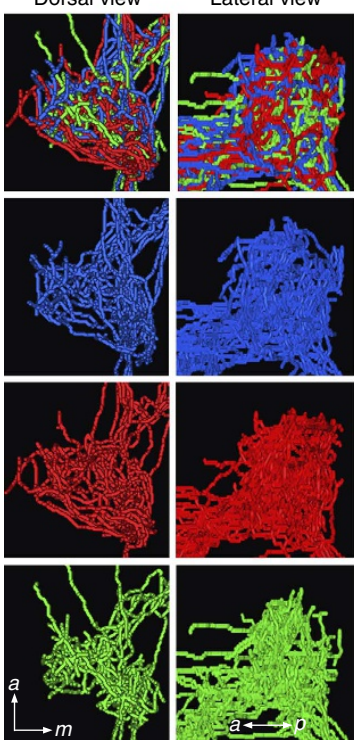

C

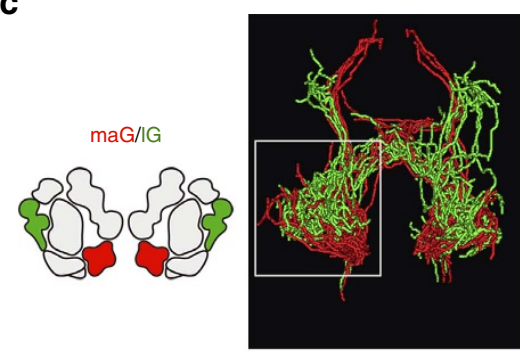

Dorsal view Lateral view
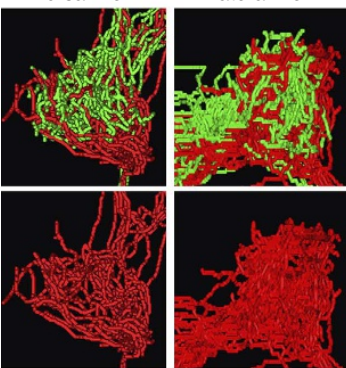

Caudal view
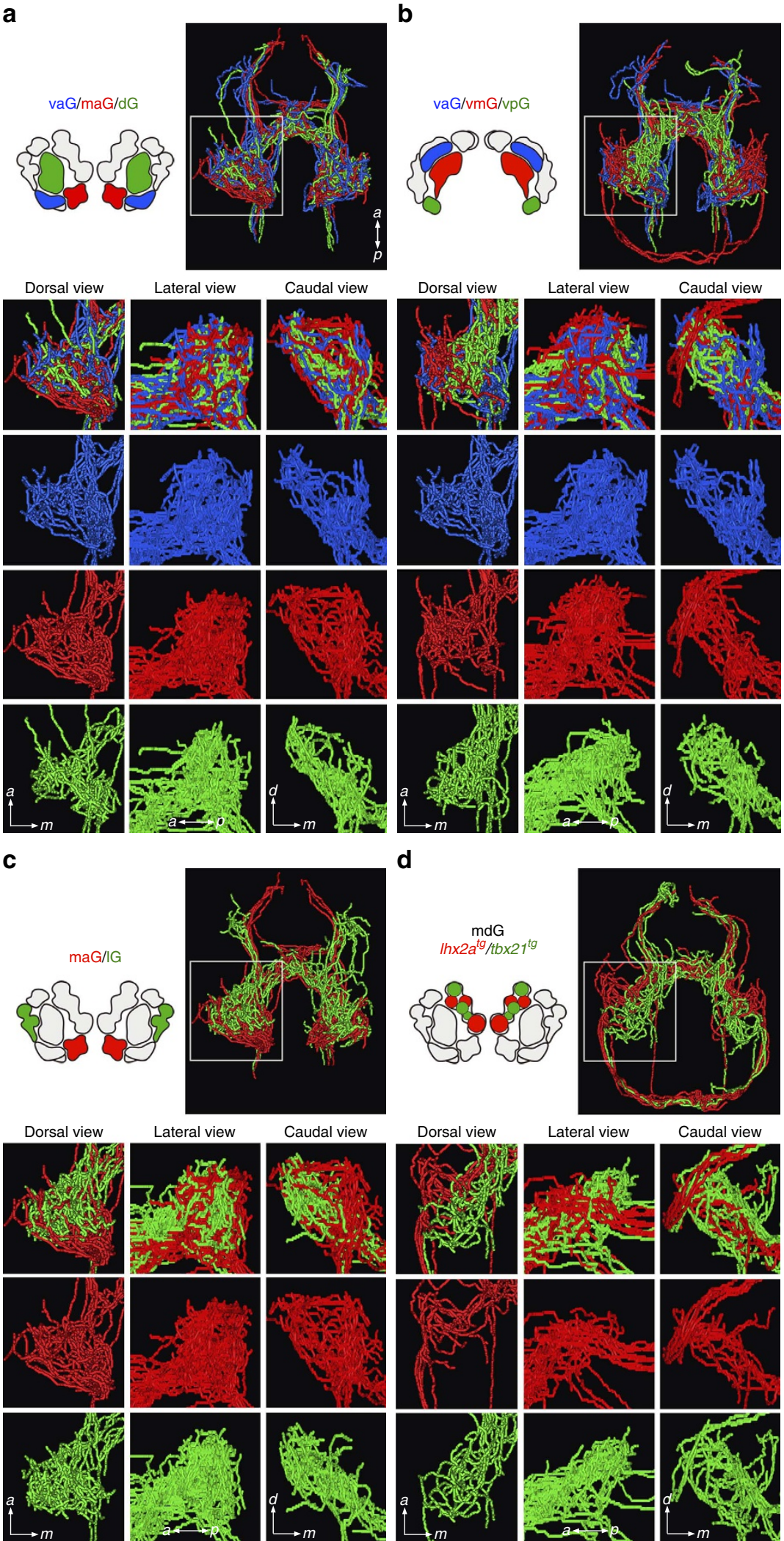

Caudal view

d

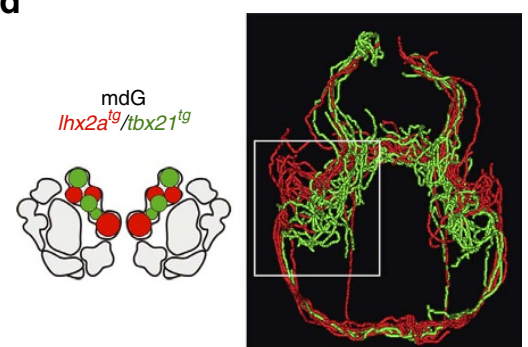

Dorsal view Lateral view Caudal view
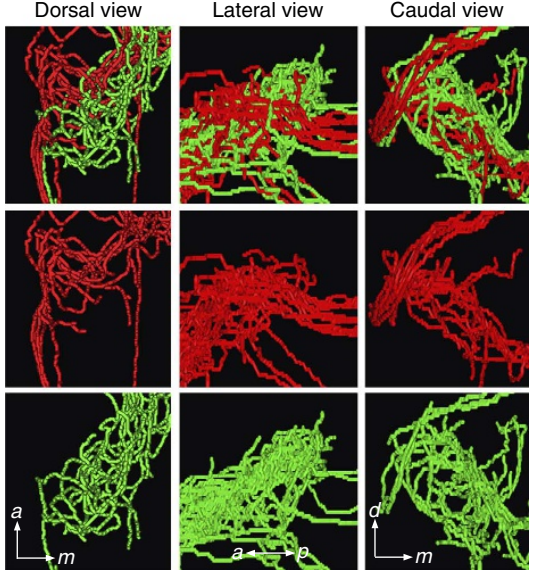

Figure 4 | Spatial distributions of axon projections from different classes of OB output neurons in the pTel. (a-d) Three-dimensional renderings of axon tracings in the forebrain with different combinations of OB output neuron classes are shown in the dorsal view (top right panels). The tracings within the pTel (box) are shown in the dorsal, lateral and caudal views. Separate renderings for each class are shown in the lower panels. (a) vaG (blue), maG (red)

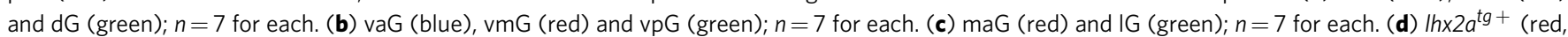
$n=7$ ) and $t b \times 21^{t g}+$ (green, $n=6$ ) output neurons innervating different sets of glomeruli within mdG. 

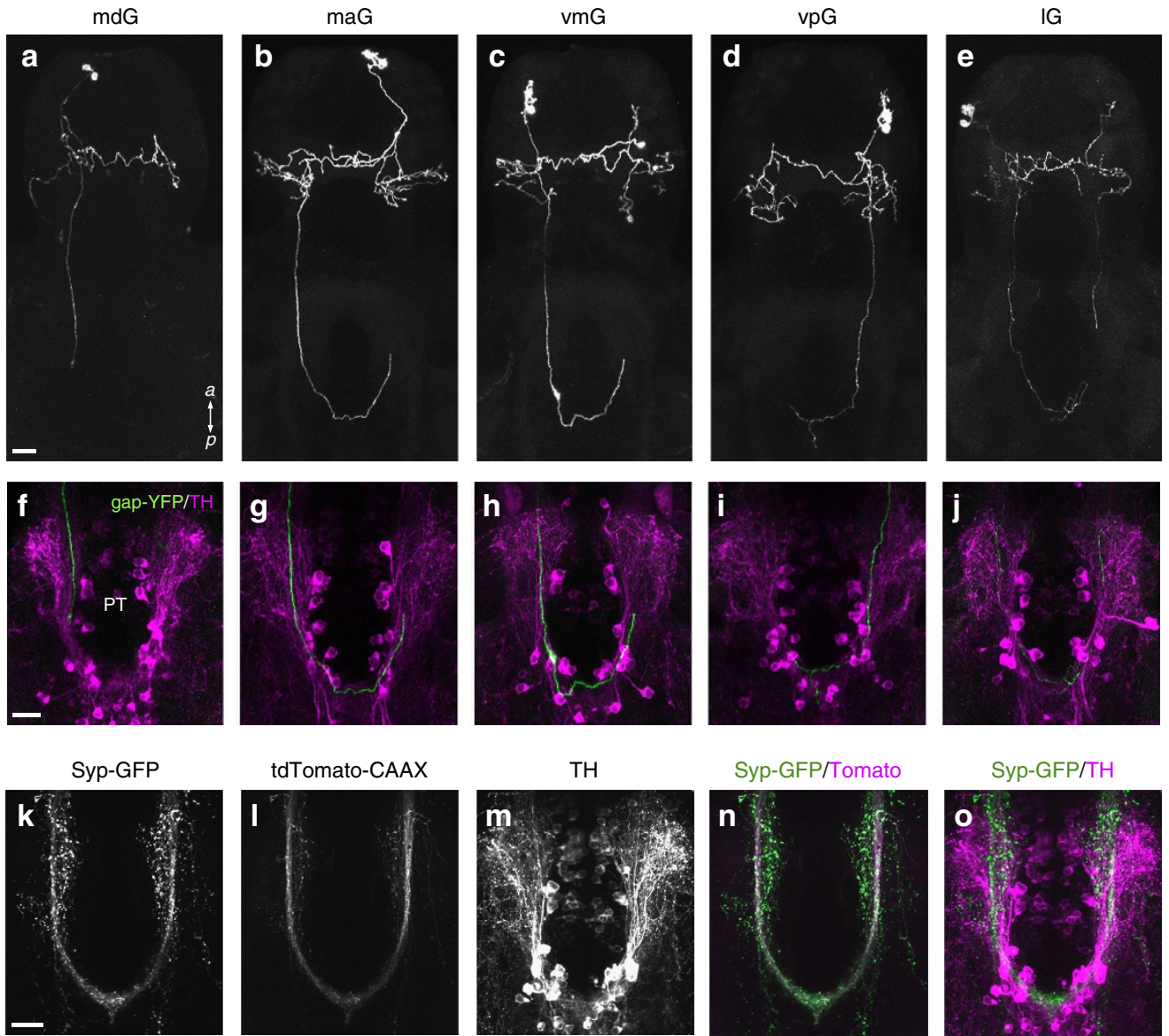

tdTomato-CAAX
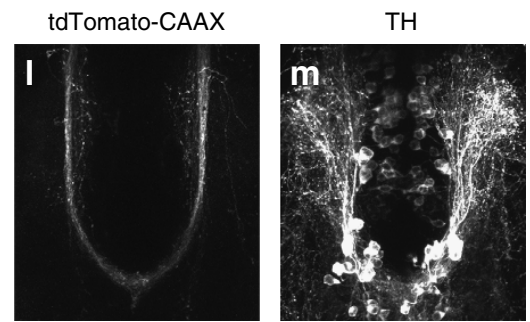

Syp-GFP/Tomato

\section{Syp-GFP/TH}
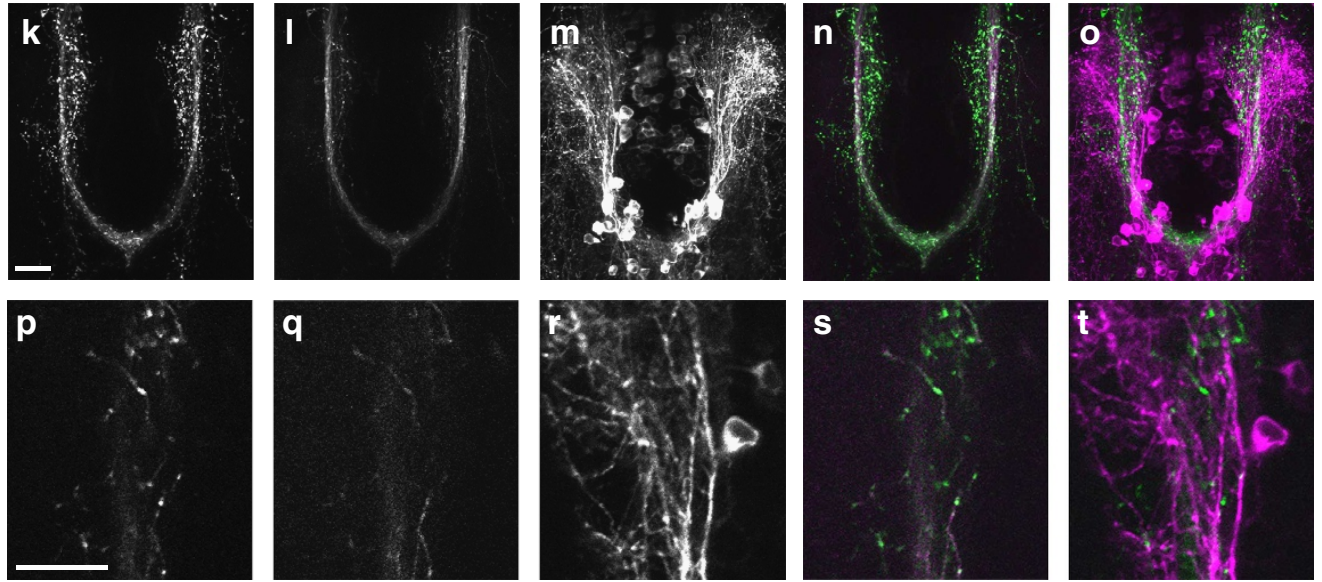

Figure 5 | The majority of $\mathbf{t} b \times 21^{\mathbf{t g}}+\mathbf{O B}$ output neurons display axon projections to the PT. (a-e) Confocal Z-stack images of five representative tbx2 ${ }^{t g}+$ $\mathrm{OB}$ output neurons innervating different glomerular clusters ( $\mathrm{mdG}, \mathrm{maG}, \mathrm{vmG}, \mathrm{vpG}$ and $\mathrm{IG}$ ) are shown in the ventral view. (f-j) Axons (green) extending from the $\mathrm{OB}$ are closely associated with tyrosine hydroxylase (TH)-immunoreactive processes (magenta) elaborated by dopaminergic neurons in the PT. (k-o) Confocal Z-stack images of the PT in a transgenic larva carrying tbx21:Gal4 and UAS:Syp-GFP-T2A-tdTomato-CAAX transgenes. The whole-mount brain is stained with anti-GFP ( $\mathbf{k}$; green in $\mathbf{n}$ and $\mathbf{o}$ ), anti-DsRed (I; magenta in $\mathbf{n}$ ) and anti-TH ( $\mathbf{m}$; magenta in $\mathbf{o}$ ) antibodies and the PT is shown in the ventral view. (p-t) Selected single-optical section with higher magnification. Some of Syp-GFP puncta are observed in close proximity to TH-positive processes (t). Scale bar, $20 \mu \mathrm{m}$.

position (Figs $2 \mathrm{~b}$ and $6 \mathrm{a}) \cdot \mathrm{tb} \times 21^{\mathrm{tg}+}$ output neurons innervating mdG (44\% for ipsilateral; $56 \%$ for contralateral), vpG (10\%; 40\%), vmG $(18 \% ; 27 \%)$ and $\mathrm{lG}(27 \% ; 36 \%)$ were the major contributors to the intra/inter-bulbar projections. The axons extending back into the ipsilateral $\mathrm{OB}$ often terminated in the proximity of the labelled soma (Fig. 6a, mdG and dG; Fig. 6b, left). Notably, the axons entering the contralateral $\mathrm{OB}$ appeared to terminate near the corresponding position equivalent to its soma origin in the ipsilateral OB (Fig. 6a, mdG, vmG and lG; Fig. 6b, middle and right). We quantitatively assessed whether the projections to ipsi- and contralateral OBs are topographically organized. We first mirrored somata from one side to the other side of OBs (see Methods), and then measured distances from individual axon terminals to all somata including both the mirrored and unmirrored ones within the same bulb. In the case of projections to the contralateral $\mathrm{OB}$, the mirrored soma and its original axon terminal(s) are defined as intra-neuronal pair. Cumulative distributions of distances for intra-neuronal soma/terminal pairs in ipsilateral $(n=12)$ and contralateral $(n=26)$ projections deviated from those for interneuronal pairs (ipsi, $n=84$; contra, $n=286$ ) towards shorter distances $(P=0.034$ for ipsi; $P=0.048$ for contra; KolmogorovSmirnov test, two-sided) (Fig. 6c,d), indicating coarse topography of projections to the OBs. These results suggest that the projections to ipsilateral and contralateral OBs may play functional roles in generating feedback connections to the originating glomerular units and coordinating the isofunctional glomerular units between the left and right OBs, respectively.

Diverse patterns of axon branching from the same glomerulus. Finally we asked whether OB output neurons innervating the same glomerulus display a stereotypical axon branching pattern, as was indicated for output neurons of the Drosophila antennal lobe (OB equivalent $)^{16,17}$. Zebrafish larvae possess 10 large, identifiable 
a
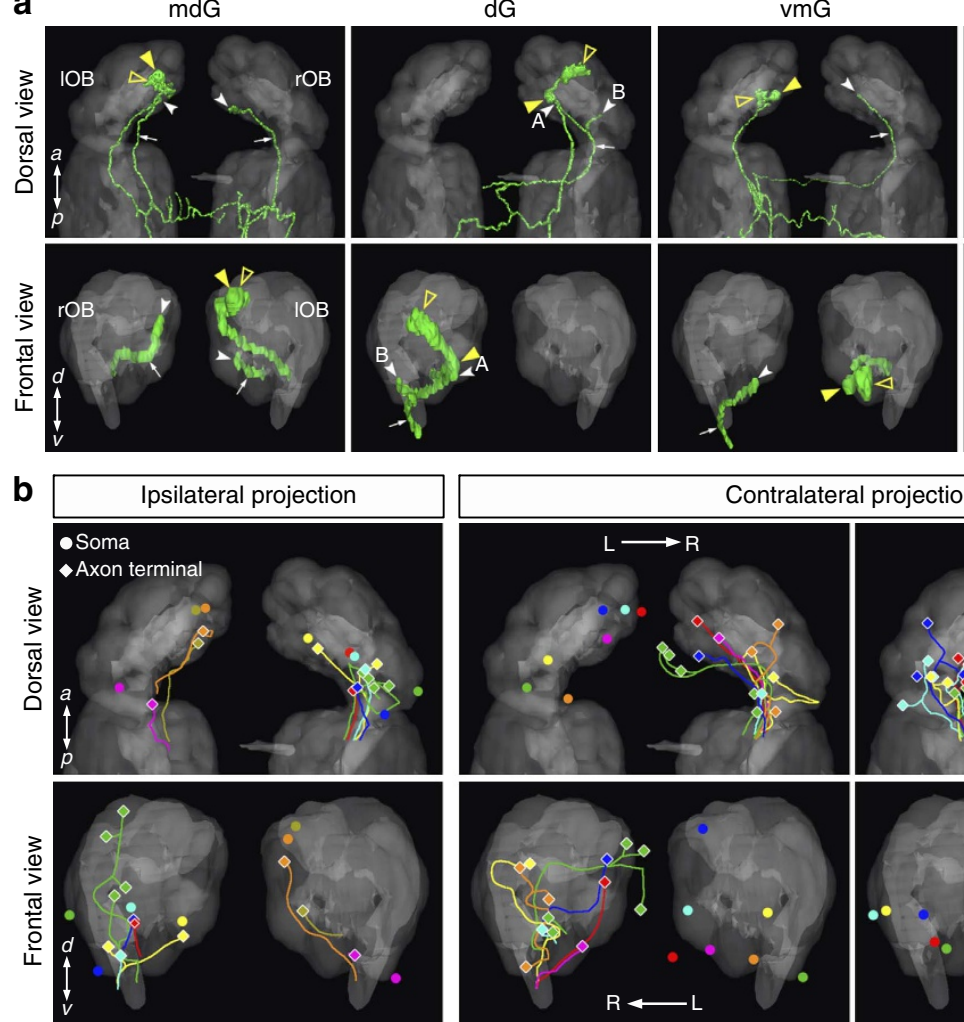

C

Ipsilateral projection

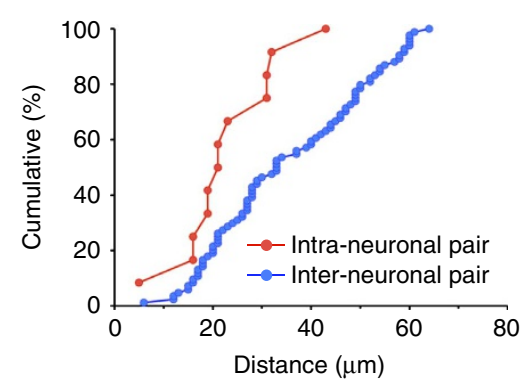

d

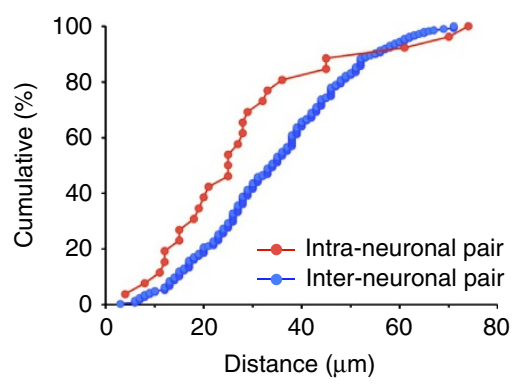

Contralateral projection
IG
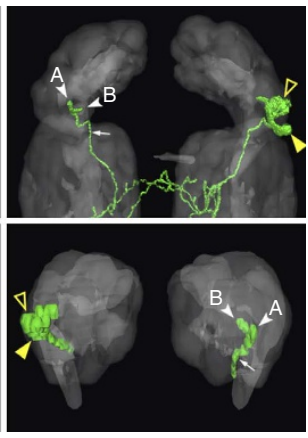

al projection
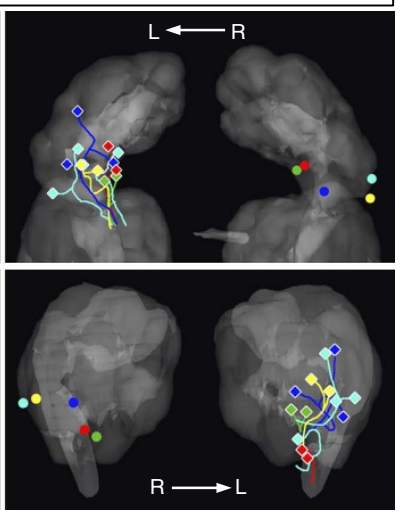

Figure 6 | Intra- and inter-bulbar projections are coarsely topographic. (a) Four representative neurons displaying projections to the ipsilateral and/or contralateral $\mathrm{OB}$ are shown onto the reference brain (grey) in the dorsal (upper panels) and frontal (lower panels) views. Somata (closed yellow arrowheads), dendrites (open yellow arrowheads), recurrent axon shafts (arrows) and axon terminals (white arrowheads) are indicated. (b) Schematic representations of eight neurons ( $2 \mathrm{mdG}, 2 \mathrm{dG}, 1 \mathrm{vaG}, 1 \mathrm{vpG}$ and $2 \mathrm{IG}$ ) for ipsilateral projection and twelve neurons ( $2 \mathrm{mdG}, 1 \mathrm{maG}, 1 \mathrm{vaG}, 3 \mathrm{vmG}, 2 \mathrm{vpG}$ and 3 IG) for contralateral projection in the dorsal (upper panels) and frontal (lower panels) views. Circles, lines and diamonds represent somata, axon trajectories and axon terminals, respectively. (c,d) Cumulative distribution plots for the distances between somata and axon terminals of neurons displaying ipsilateral projection (c) and contralateral projection (d). The distributions for intra-neuronal soma/terminal pairs (red; ipsilateral, $n=12 ;$ contralateral, $n=26$ ) deviate from those for inter-neuronal pairs (blue; ipsi, $n=84$; contra, $n=286)$ towards shorter distances $(P=0.034$ for ipsi; $P=0.048$ for contra; Kolmogorov-Smirnov test, two-sided).

glomeruli that emerge during embryonic development and persist in stable configurations afterwards ${ }^{23}$; these include six glomeruli in mdG ( $\left.\mathrm{mdG}_{1-6}\right)$, two in $\mathrm{lG}\left(\mathrm{lG}_{3,4}\right)$ and two in vpG $\left(\mathrm{vpG}_{1,2}\right)$. Individual glomeruli other than the 10 identifiable ones cannot readily be discerned by SV2 staining at early larval stages ${ }^{23}$, but we noticed that dendritic tufts of some output neurons shared and occupied a restricted area within the glomerular clusters: lateroventral and lateral portions of $\mathrm{maG}$ (maG-lv and $\mathrm{maG}-l)$. We therefore picked out output neurons innervating six of the ten large glomeruli in addition to $\mathrm{maG}-l v$ and $\mathrm{maG}-l$. Axons from an identical glomerulus displayed a defined target region in the pTel, but no stereotypical pattern of branching within that region (Fig. 7b-i; Supplementary Fig. 6). Whether or not axon branches target other forebrain areas (vTel, rHb, PT and OB) was not necessarily invariable for output neurons innervating the same glomerulus. Furthermore, axon branches that project to the $\mathrm{rHb}$ or PT did not always exit the same hemisphere of the telencephalon. We also encountered a case where one $l h \times 2 a^{t g}+$ neuron and one $t b \times 21^{t g}+$ neuron innervate the same glomerulus, mdG $_{1}$ (Fig. 7a; Supplementary Fig. 6a). Dendritic tufts of these neurons appeared to segregate into different compartments within the $\mathrm{mdG}_{1}$, and their axon branching patterns in the pTel as well as the target choice outside the telencephalon were completely different.

\section{Discussion}

To the best of our knowledge, this is the first study describing a nearly comprehensive projection map of the secondary olfactory 
a
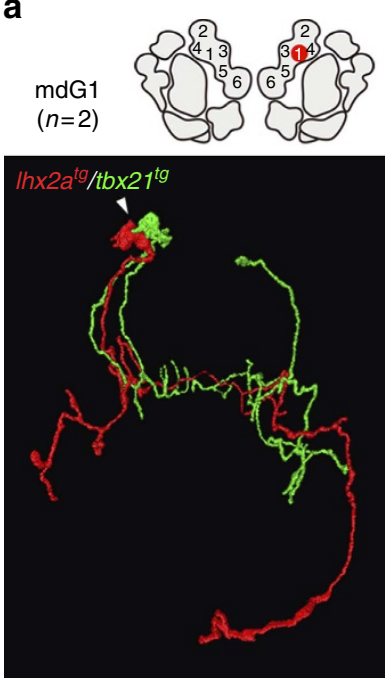

d
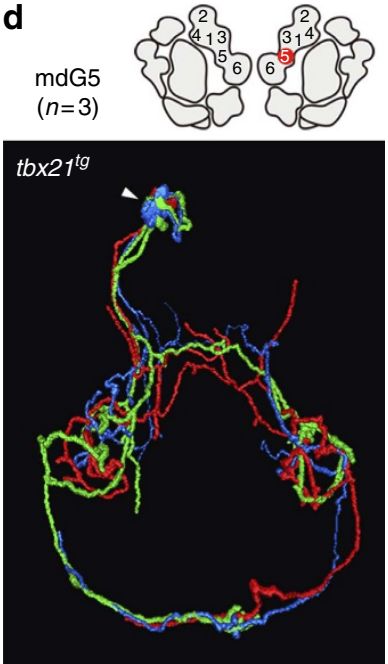

g
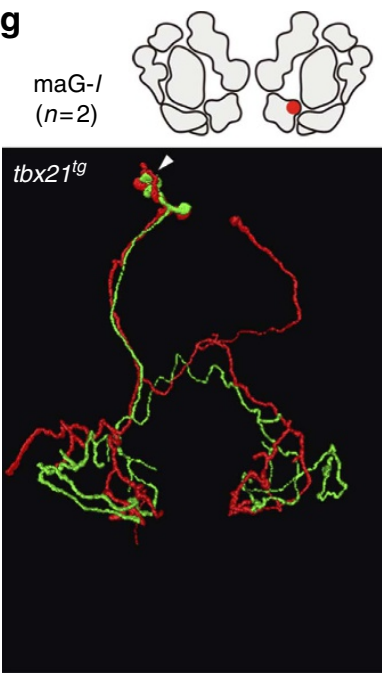

b

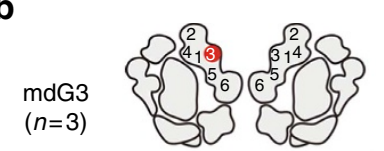

$\ln \times 2 a^{\text {tg }}$

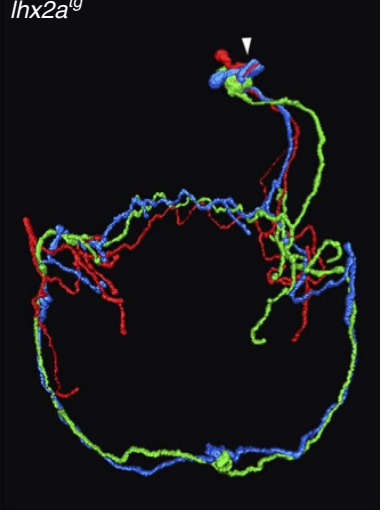

e

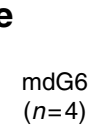

Ihx $2 a^{\text {tg }}$
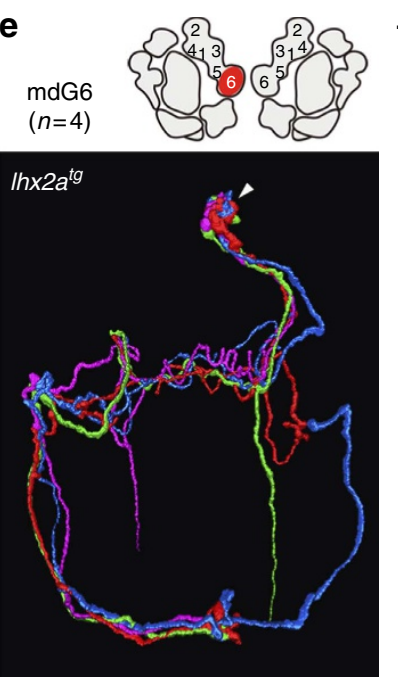

$\mathbf{h}$

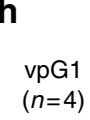

$t b \times 21^{\text {tg }}$

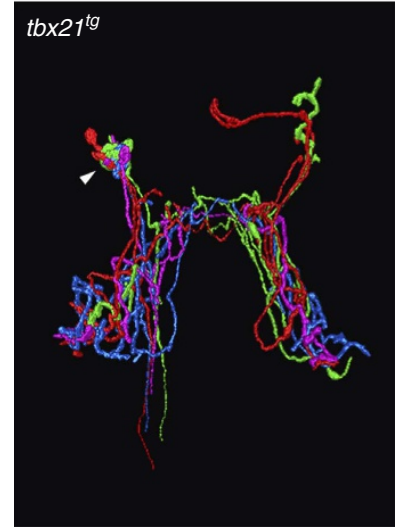

C
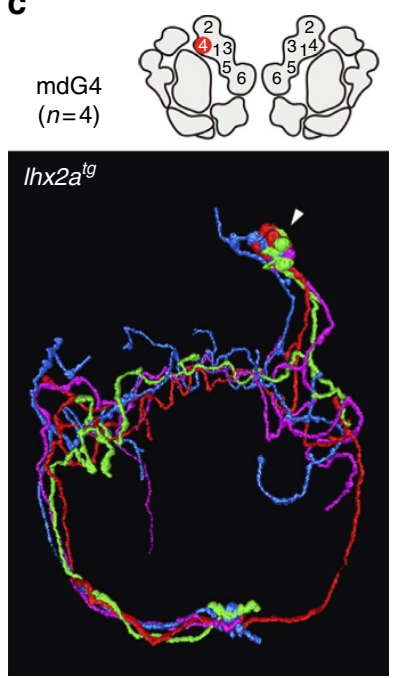

\section{f}

maG-lv $(n=4)$
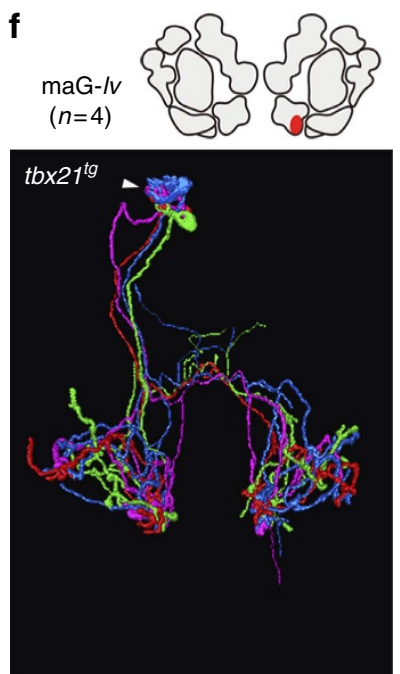

i
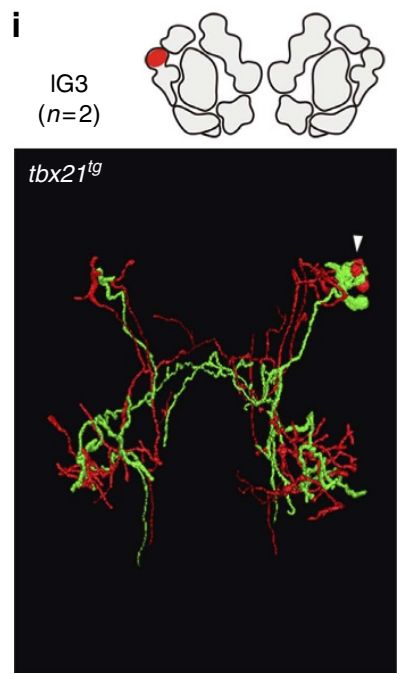

Figure 7 | Diverse patterns of axon branching of OB output neurons innervating the same glomerulus. (a-i) Three-dimensional reconstructions of two to four output neurons innervating the same glomerulus are simultaneously visualized in the dorsal view. Different colours indicate different neurons. Arrowhead in each panel denotes the position of glomerulus innervated. Projection patterns of individual output neurons are shown in Supplementary Fig. 6.

pathway at single-axon resolution in vertebrates. Five major target regions, the pTel, vTel, $\mathrm{rHb}, \mathrm{PT}$ and $\mathrm{OB}$ itself, receive different patterns of projections with respect to spatial organization (diffuse or convergent) as well as originating glomerular classes (non- selective or biased) (Fig. 8). These findings help decipher how odour information is translated in higher brain centres.

The present study reveals two major types of glomerular representations in the telencephalon: non-selective versus biased. 

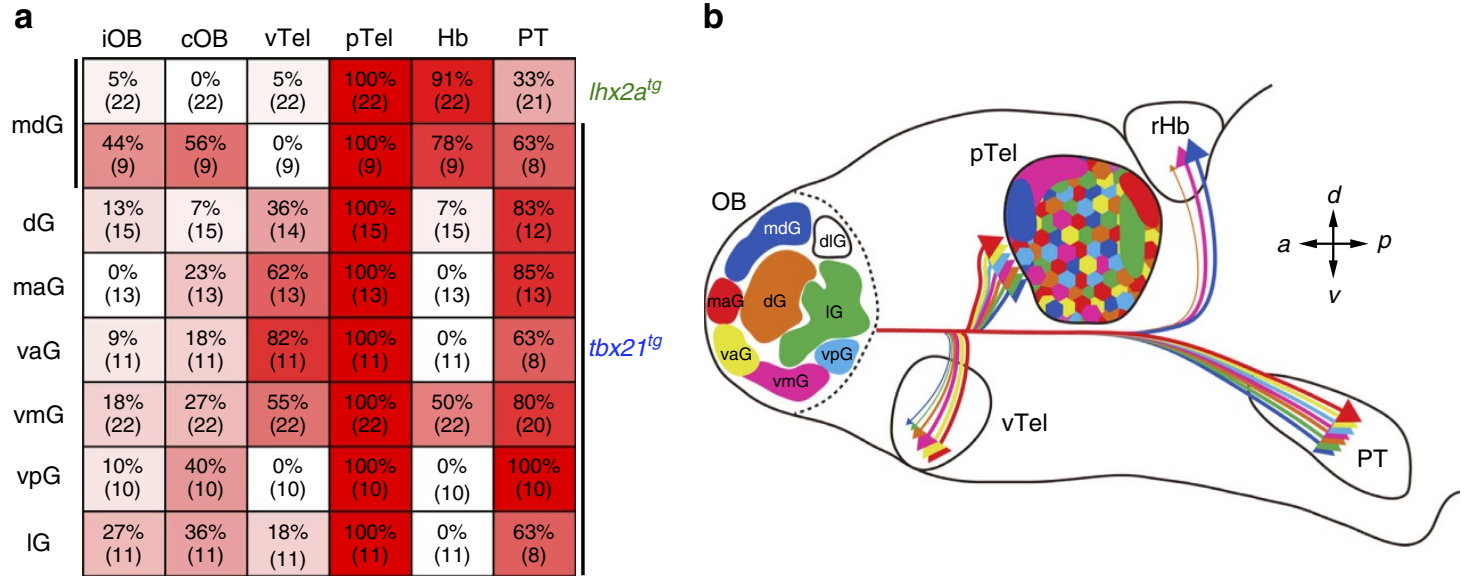

Figure 8 | Overview of axon projections from the OB to the forebrain regions. (a) Axon projections to the forebrain target regions (columns) from each glomerular cluster (rows) are indicated quantitatively by the percentage of neurons projecting to the region per analysed neurons. Ihx $2 a^{t g}+$ and tbx $27^{t g}+$ output neurons innervating $\mathrm{mdG}$ are indicated separately. Gradations in red colour for boxes also represent the percentages. Numbers of neurons analysed for each projection are given in parentheses. This analysis includes larvae displaying reversed laterality of the habenulae. iOB, ipsilateral olfactory bulb; $\mathrm{COB}$, contralateral olfactory bulb; vTel, ventral telencephalon; pTel, posterior telencephalon; Hb, habenula; PT, posterior tuberculum. (b) Schematic overview of axon projections from the $\mathrm{OB}$ to forebrain regions other than the $\mathrm{OB}$ itself in 7-d.p.f. zebrafish larvae. Projection pathways to the target regions are depicted by arrows in lateral view, and the frequency of projections are represented as the size of arrows. The central portion of pTel receives distributive and overlapping projections from all glomerular clusters examined, whereas the $\mathrm{vTel}$, $\mathrm{rHb}$, and peripheral portions of the pTel receive biased projections from particular glomerular clusters. The PT receives convergent projections from all glomerular clusters examined.

The central portion of the pTel receives distributive projections from all the glomerular clusters. In addition, diversity of axon branching patterns is observed for output neurons innervating the same glomerulus, suggesting stochastic innervation of neurons in this area. A previous optical imaging study ${ }^{39}$ in adult zebrafish demonstrated that different classes of odorants activating distinct glomerular clusters in the $\mathrm{OB}$ evoke responses of unique ensembles of neurons dispersed and intermingled in the $\mathrm{Dp}$. The $\mathrm{Dp}$ is a pallial structure homologous to the olfactory cortex $^{40}$ and arises from the larval pTel. Thus, the distributive and overlapping projections from all the glomerular clusters to the pTel would serve as an anatomical substrate for interspersed odour representations in the $\mathrm{Dp}$. In contrast to the central portion of the $\mathrm{pTel}$, its marginal portions and the vTel receive biased inputs from particular glomerular clusters. In channel catfish, telencephalic areas that preferentially respond to specific classes of biologically relevant odorants have been reported ${ }^{41}$. Further functional studies will clarify whether there still remains the spatial organization of odour responsiveness in the Tel of zebrafish.

A remarkable feature of the circuitry we identified is the direct projections from the $\mathrm{OB}$ to the two diencephalic nuclei, $\mathrm{rHb}$ and $\mathrm{PT}$. The $\mathrm{Hb}$ constitutes the dorsal diencephalic conduction pathway that connects limbic forebrain to brainstem nuclei including the interpeduncular nucleus ${ }^{42}$. In zebrafish, the $\mathrm{Hb}$ is anatomically and molecularly divided into the dorsal and ventral regions $^{43}$, and the dorsal habenulae exhibit a prominent left-right asymmetry in the size ratio of medial and lateral subnuclei, which innervates the ventral and dorsal parts of the interpeduncular nucleus, respectively ${ }^{44}$. Axons of $\mathrm{OB}$ output neurons terminate at the medialmost part of the neuropil domain in the $\mathrm{rHb}$, presumably transmitting the odour information to the ventral part of interpeduncular nucleus. Two recent studies in zebrafish have shown that the $\mathrm{Hb}$ plays roles in controlling fear responses in experience-dependent manners ${ }^{45,46}$. However, it is tempting to speculate that the OB-rHb pathway may be a part of hard-wired circuits that mediate stereotyped responses such as innate behaviours, because the $\mathrm{rHb}$ receives strongly biased projections from particular glomerular clusters. Thus, it will be of interest to elucidate whether the $\mathrm{Hb}$ could be involved in regulating not only learned behaviours but also innate behaviours.

In contrast to the $\mathrm{rHb}$, the $\mathrm{PT}$ receives convergent projections from all the glomerular clusters, suggesting a wide range of responsiveness of $\mathrm{PT}$ neurons to various odour stimuli. We find that the axons and their synaptic terminals of $\mathrm{OB}$ output neurons are closely associated with processes of dopaminergic neurons in the PT, rendering them a strong candidate for postsynaptic partner. Because a population of dopaminergic neurons in the PT send descending axons directly to the spinal $\operatorname{cord}^{37}$, it is conceivable that the OB-PT pathway may play general roles in olfaction such as evoking locomotion ${ }^{47,48}$. Further studies are required to precisely identify types of PT neurons connecting with the $\mathrm{OB}$ output neurons and to elucidate the functional aspects of this unique neural circuitry.

The olfactory circuits we describe in zebrafish provide insights into anatomical similarity and dissimilarity with those of insects and mammals, especially with Drosophila and mouse. In all the three species, individual output neurons in the $\mathrm{OB}$ or the antennal lobe send axons to multiple brain areas. Each brain area appears to adopt one of two major strategies of projections to be received: (1) restricted and stereotyped projections with respect to glomerular classes for the fly lateral horn ${ }^{14}$, zebrafish $\mathrm{Hb}$ and mouse cortical amygdala ${ }^{9,10} ;(2)$ broad and random projections for the fly mushroom body ${ }^{49}$, zebrafish pTel (Dp), and mouse piriform cortex ${ }^{9,10}$. Thus, our findings support the idea that the two distinct modes of connectivity patterns (stereotyped and random), which are suitable for innate and learned behaviours, are applicable to all animal species as a fundamental principle for olfactory representations in higher brain centres. As to the axon branching of output neurons innervating the same glomerulus, highly diverse patterns are observed in zebrafish and mouse, compared with more stereotyped one in Drosophila ${ }^{16,17}$. Furthermore, we find in zebrafish that choice of target areas in the forebrain by output neurons innervating the same glomerulus is not always the same. Thus, the increased diversity of projections in vertebrates may serve to enhance the ability to perform combinatorial processing of odour information beyond the OB. 
Another interesting point to compare is what order of olfactory circuits first conveys odour information to the contralateral brain hemisphere. The first-, second- and third-order neurons send axons contralaterally in Drosophila, zebrafish and mouse, respectively. Although individual OSNs in Drosophila project axons bilaterally to both sides of the antennal lobe, asymmetric transmitter release enables flies to navigate towards the odour source $^{50}$. In mouse, the third-order neurons in the anterior olfactory nucleus connect both hemispheres and play a role in detecting a localized odour source ${ }^{51}$. Maintaining the ipsilateral projections up to higher order circuitry may enhance lateralization of odour information, whereas the contralateral projection at lower order circuitry may increase the capacity of information processing and/or provide useful redundancy as a reserve of almost equivalent sensory pathway. Thus, individual animal species may evolve to optimize the olfactory circuits based on the trade-off between those benefits, which can be affected by the size of brain. However, it remains possible that the difference may also account for characteristic behaviour of each species. Further functional studies of the olfactory circuits described in this study will provide not only general principles in decoding of odour information but also evolutional insights into the circuitry relevant to species-specific behaviours.

\section{Methods}

Animals. Zebrafish, Danio rerio, were maintained at $28.5^{\circ} \mathrm{C}$ on a 14 -h light $/ 10$-h dark cycle and embryos were collected by natural spawning. Embryos for imaging experiments were treated with $0.002 \% N$-phenylthiourea starting at $12-14 \mathrm{~h}$ postfertilization to inhibit pigment formation. All experimental procedures were approved by the Animal Care and Use Committee of RIKEN.

DNA constructs. To generate $B A C-t b x 21: z G F F$ transgene (referred to as tbx21:Gal4), a bacterial artificial chromosome (BAC) that contains a zebrafish genomic DNA fragment ( $\sim 200 \mathrm{~kb}$ in length; clone CH211-194K22) encompassing the $t b \times 21$ gene was modified by RedET-mediated homologous recombination in Escherichia coli (Counter-selection kit, Gene Bridges). The region from the translation initiation codon of $t b \times 21$ gene to the end of the first coding exon was replaced with a modified Gal4 cDNA (Gal4FF optimized for a zebrafish codon usage, a gift from Koichi Kawakami, National Institute of Genetics, Japan), followed by an SV40 polyadenylation sequence. To generate UAS:tdTomato-CAAX transgene, the $t d$ Tomato cDNA was PCR-amplified from $p C S 2$-tdTomato-2A-GFP (a gift from Shankar Srinivas, University Oxford) and fused in frame with a sequence encoding CAAX box, a membrane-targeting motif. The resulting $t d T o$ mato-CAAX cDNA was then inserted between BglII and XhoI sites of pT2MUASMCS (a gift from Koichi Kawakami) which contains five tandem repeats of Gal4-binding sequence (UAS), a TATA sequence, an SV40 polyadenylation sequence and two Tol2 transposon elements. To generate UAS:DsRed-Express transgene, the DsRed-Express cDNA (Clontech) was inserted between BglII and XhoI sites of the $p$ T2MUASMCS. To generate UAS:Syp-GFP-T2A-tdTomato-CAAX transgene, Syp-GFP cDNA (a gift from Stephen J. Smith, Stanford University) and tdTomato-CAAX cDNA were amplified and fused in frame via a sequence encoding the Thosea asigna virus $2 \mathrm{~A}$ peptide (T2A) by recombinant PCR. The primers used are as follows: Syp-GFP forward, $5^{\prime}$-GGA TCC GCC ACC ATG GAT GTT GCC AAC CAG TTG GTC GCC ACT G-3'; Syp-GFP reverse (including the T2A antisense sequence), 5' ${ }^{\prime}$ TGG GCC AGG ATT CTC CTC GAC GTC ACC GCA TGT TAG CAG ACT TCC TCT GCC CTC TCC ACT GCC CTT GTA CAG CTC GTC CAT GCC GAG-3'; tdTomato-CAAX forward (including the T2A sense sequence), 5'-GGC AGT GGA GAG GGC AGA GGA AGT CTG CTA ACA TGC GGT GAC GTC GAG GAG AAT CCT GGC CCA ATG GTG AGC AAG GGC GAG GAG GTC- $3^{\prime}$; $t$ t Tomato-CAAX reverse, $5^{\prime}$-ACG CGT GGG CCC TCT AGA GGC TCG AGT TAC ATG ATC ACG CAC TTG GTC TTG C-3'. The resultant Syp-GFP-T2A-tdTomato-CAAX cassette was inserted between EcoRI and XhoI sites of the pT2MUASMCS. plhx2a:Gal4-VP16 and pUAS:gap-YFP were generated in our previous study ${ }^{20}$.

Generation of transgenic zebrafish lines. To generate $\operatorname{TgBAC}(\operatorname{tb} \times 21: z G F F)$ transgenic line, BAC DNA $\left(10 \mathrm{ng}^{-1} \mathrm{l}^{-1}\right)$ was co-injected with PI-SceI meganuclease $\left(1 \mathrm{U} \mathrm{ml}^{-1}\right.$, New England Biolabs) into one-cell-stage embryos. The resultant fish were crossed with homozygous $\mathrm{Tg}\left(U A S: G F P\right.$ ) reporter fish ${ }^{52}$ (a gift from Koichi Kawakami). An $\mathrm{F}_{1}$ embryo carrying both BAC-tbx21:zGFF and UAS:GFP transgenes was identified with GFP fluorescence and raised to establish the Gal4 driver line. To generate $\operatorname{Tg}$ (UAS:tdTomato-CAAX), $T g(U A S: D s R e d$-Express) and Tg(UAS:Syp-GFP-T2A-tdTomato-CAAX) reporter lines, plasmid DNA

$\left(20{\mathrm{ng} \mathrm{l}^{-1}}^{-1}\right)$ was co-injected into one-cell-stage embryos with Tol2 transposase
mRNA (20 ng $\mathrm{ll}^{-1}$ ) that was synthesized in vitro (mMESSAGE mMACHINE T7 Ultra Kit, Ambion). The resultant fish were crossed with transgenic fish carrying the BAC-tbx21:zGFF transgene. Among the offspring, embryos that showed brightest fluorescence of tdTomato-CAAX, DsRed-Express or Syp-GFP were raised to establish the reporter lines. Two transgenic zebrafish lines, $\operatorname{Tg}(\operatorname{lh} \times 2 a: G F P)$ and $\operatorname{Tg}(\operatorname{lh} \times 2 a: g a p-Y F P)$, were generated in our previous study ${ }^{20}$.

Genetic single-neuron labelling. A mixture of two DNA constructs, $B A C$

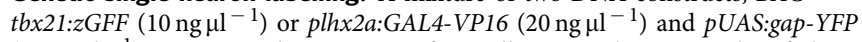
$\left(10 \mathrm{ng} \mu \mathrm{l}^{-1}\right)$, was injected into one- to four-cell-stage embryos. To identify larvae with single-labelled neurons, the injected larvae were screened at 3 d.p.f. and again at 7 d.p.f. under an inverted microscope (Olympus IX81) equipped with FV500 confocal laser-scanning system through $\mathrm{x} 40$ water-immersion objective lens (NA 0.9, Olympus PlanApo). The 7-d.p.f. larvae harbouring single-labelled neurons were then fixed in $10 \%$ formalin (3.7\% formaldehyde, $0.8 \%$ methanol) in phosphate-buffered saline (PBS) for $1-1.5 \mathrm{~h}$ at room temperature. After washing with PBS containing 0.5\% Tween-20 (PBSTw), the brains were dissected out with fine forceps and stored in PBSTw at $4{ }^{\circ} \mathrm{C}$ until use for further immunohistochemical analyses.

Immunohistochemistry. The fixed brains of 7-d.p.f. larvae were blocked by $10 \%$ normal goat serum (NGS) in PBS containing $2 \%$ dimethylsulphoxide and $0.25 \%$ Triton X-100 (PBDTx) overnight at $4{ }^{\circ} \mathrm{C}$. The brains were incubated with primary antibodies in 5\% NGS/PBDTx for 3-7 days at $4{ }^{\circ} \mathrm{C}$, washed thoroughly over a day in PBDTx at room temperature and then incubated with appropriate secondary antibodies in $5 \%$ NGS/PBDTx for $1-3$ days at $4{ }^{\circ} \mathrm{C}$. After washing thoroughly with PBDTx, the brains were mounted in $2 \%$ low melting-point agarose gel on glass coverslips for confocal imaging. The following antibodies were used: rat monoclonal anti-GFP antibody (1:1,000, Nacalai Tesque); rabbit polyclonal anti-RFP antibody (1:1,000, Abcam); rabbit polyclonal anti-DsRed antibody (1:500, Clontech); mouse monoclonal anti-SV2 antibody (1:1,000, Developmental Studies Hybridoma Bank); rabbit polyclonal anti-TH antibody (1:200, Millipore); mouse monoclonal anti-TH antibody (1:400, Millipore); Alexa488-conjugated donkey anti-rat IgG antibody (1:300, Molecular Probes); CF-555-conjugated donkey anti-rabbit IgG antibody (1:300, Biotium); CF633-conjugated donkey anti-mouse IgG antibody (1:300, Biotium).

Image acquisition. Brains were scanned every $1 \mu \mathrm{m}$ using an inverted microscope (Olympus IX81) equipped with FV1000 confocal laser-scanning system. Imaging parameters used for (1) stable transgenic larvae and (2) single-cell labelling experiments were the following: (1) x40 water-immersion objective lens (NA 0.9, Olympus PlanApo), $512 \times 512$ pixels with $\times 2.0$ zoom (frontal view) or x1.3 zoom (dorsal view), $1024 \times 480$ pixels with x1.0 zoom (ventral view), $640 \times 640$ pixels with $\times 2.0$ zoom (close-up view of the PT); (2) x30 silicone oil-immersion objective lens (NA 1.05, Olympus UPLSAPO), $512 \times 512$ pixels with x1.7 zoom (dorsal view), $640 \times 480$ pixels with x1.0 zoom (ventral view).

Image registration. Image registration was carried out using elastix ${ }^{29}$, an intensitybased 3D image registration software. Brain images were aligned to a reference brain using an initial affine registration, followed by a non-rigid registration (elastic transform represented with B-splines) with Mattes mutual information as the similarity measure ${ }^{53}$. The final control point grid of B-spline coefficients had a mean spacing of $5 \mu \mathrm{m}$ (image size: $249.344 \mu \mathrm{m} \times 249.344 \mu \mathrm{m} \times 167 \mu \mathrm{m}$; number of control points: $49 \times 49 \times 33$ ). We aligned 63 brains that display bright SV2 staining and normal laterality of the habenulae. To create the reference brain, 20 brain images were registered to a selected brain image (seed brain), which had high signal to noise of SV2 staining and largest number of optical slices (167 slices), and then the registered brain images together with the seed brain were averaged using the open-source toolbox Fiji ${ }^{5}$. Next, the SV2 channel of each individual brain was registered to the reference brain. The resulting transformation for the SV2 channel was then applied to the image channel containing a single-labelled neuron using transformix ${ }^{29}$.

Validation of registration accuracy. Registration was first evaluated based on the similarity metric value and then through visual inspection in Fiji by superimposing the deformed SV2 images on the reference SV2 image. A deformed image was judged to be successful if the external boundary and internal structure of SV2 staining matched the reference. The Tel in all the deformed brain images (63 brains) matched the reference well, although the external boundary of the $\mathrm{Hb}$ in 14 out of 63 deformed brains showed a slight deviation from that of reference brain, presumably due to higher morphological variability of the $\mathrm{Hb}$ across individuals. We adopted all 63 registered images for further processing and analyses because we especially focused on projection patterns within the Tel in this study. We also quantitatively assessed the registration errors that could be caused by variation of sample orientations. We imaged the same brain with two different orientations (dorsal-oblique views), registered them to the reference brain, and evaluated the variance of two registered neurons (Supplementary Fig. 3). Mounting angle relative to the vertical midline of the reference brain was set between 10 and 15 degrees for 
each side. The registration error was evaluated by measuring the distance between 15 corresponding 3D landmarks within the Tel of two registered neurons, which include axon branching points, terminals and boutons. The overall average distance measured from five brains was $2.21 \mu \mathrm{m}$ with a range of $1.74-2.62 \mu \mathrm{m}$

(Supplementary Fig. 3d). Because the maximal deviation of sample orientations (63 brains) was actually smaller (leftward, 7.5 degrees; rightward, 8 degrees relative to the reference brain), the actual error in our dataset would be lower than that shown here.

Tracing and 3D reconstruction. After image registration, neuronal tracing was carried out semiautomatically using Simple Neurite Tracer ${ }^{55}$, a plug-in for Fiji. Morphology of the traced neurons was visualized by filling out the tracing path through the 'Fill Out' processing in Simple Neurite Tracer and then subtracting noise, autofluorescence of blood vessels and faint staining of neurons other than traced ones. Three-dimensional reconstruction of the filled neurons or the traces was performed using the ImageJ $3 \mathrm{D}$ viewer ${ }^{56}$

Cluster analysis. Seven samples each for all neuronal classes other than mdG$t b \times 21^{t g}+$ neurons were randomly chosen from the registered samples; six mdG$t b \times 21^{t g}+$ neurons were used owing to the limitation of sample size ( 55 neurons in total). The tracings of axons extending towards the ventral diencephalon were first eliminated from those of axon arbors for the 55 neurons. The resultant tracings were represented as 3D stacks ( 1 for trace voxels, 0 for background voxels) and convolved with an isotropic, 3D Gaussian blur (8 voxel s.d. in each axis) with a peak value of 1 , cropped to a $17 \times 17 \times 17$ box. A bounding box spanning both hemispheres was used to restrict the analysis to an area of the telencephalon. These 3D representations were vectorized, where each pixel corresponded to an entry (dimension) of the data vector, and used as inputs to Ward's method ${ }^{30}$ to obtain the hierarchical clustering dendrograms.

Measurement of distances between somata and axon terminals. To mirror somata from one side to the other side of OBs, the SV2 channel of the reference brain was reversed horizontally, followed by being re-registered against the original reference brain using elastix. The neuronal image channels already aligned on the reference brain were similarly reversed, and the transformation for the reversed SV2 channel was applied to them using transformix. The distances from individual axon terminals to all somata, including both the mirrored and unmirrored ones, were measured within the same bulb using the ImageJ 3D-Distance-Tool.

\section{References}

1. Mori, K. \& Sakano, H. How is the olfactory map formed and interpreted in the mammalian brain? Annu. Rev. Neurosci. 34, 467-499 (2011).

2. Friedrich, R. W. \& Korsching, S. I. Combinatorial and chemotopic odorant coding in the zebrafish olfactory bulb visualized by optical imaging. Neuron 18, 737-752 (1997)

3. Friedrich, R. W. \& Korsching, S. I. Chemotopic, combinatorial, and noncombinatorial odorant representations in the olfactory bulb revealed using a voltage-sensitive axon tracer. J. Neurosci. 18, 9977-9988 (1998).

4. Uchida, N., Takahashi, Y. K., Tanifuji, M. \& Mori, K. Odor maps in the mammalian olfactory bulb: domain organization and odorant structural features. Nat. Neurosci. 3, 1035-1043 (2000).

5. Mori, K., Takahashi, Y. K., Igarashi, K. M. \& Yamaguchi, M. Maps of odorant molecular features in the Mammalian olfactory bulb. Physiol. Rev. 86, 409-433 (2006).

6. Ghosh, S. et al. Sensory maps in the olfactory cortex defined by long-range viral tracing of single neurons. Nature 472, 217-220 (2011).

7. Igarashi, K. M. et al. Parallel mitral and tufted cell pathways route distinct odor information to different targets in the olfactory cortex. J. Neurosci. 32, 7970-7985 (2012).

8. Nagayama, S. et al. Differential axonal projection of mitral and tufted cells in the mouse main olfactory system. Front Neural Circuits 4, 120 (2010).

9. Sosulski, D. L., Bloom, M. L., Cutforth, T., Axel, R. \& Datta, S. R. Distinct representations of olfactory information in different cortical centres. Nature 472, 213-216 (2011).

10. Miyamichi, K. et al. Cortical representations of olfactory input by trans-synaptic tracing. Nature 472, 191-196 (2011).

11. Stettler, D. D. \& Axel, R. Representations of odor in the piriform cortex. Neuron 63, 854-864 (2009).

12. Choi, G. B. et al. Driving opposing behaviors with ensembles of piriform neurons. Cell 146, 1004-1015 (2011).

13. Kobayakawa, K. et al. Innate versus learned odour processing in the mouse olfactory bulb. Nature 450, 503-508 (2007).

14. Jefferis, G. S. et al. Comprehensive maps of Drosophila higher olfactory centers: spatially segregated fruit and pheromone representation. Cell 128, 1187-1203 (2007).

15. Lin, H. H., Lai, J. S., Chin, A. L., Chen, Y. C. \& Chiang, A. S. A map of olfactory representation in the Drosophila mushroom body. Cell 128, 1205-1217 (2007).
16. Marin, E. C., Jefferis, G. S., Komiyama, T., Zhu, H. \& Luo, L. Representation of the glomerular olfactory map in the Drosophila brain. Cell 109, 243-255 (2002).

17. Wong, A. M., Wang, J. W. \& Axel, R. Spatial representation of the glomerular map in the Drosophila protocerebrum. Cell 109, 229-241 (2002).

18. Friedrich, R. W., Jacobson, G. A. \& Zhu, P. Circuit neuroscience in zebrafish Curr. Biol. 20, R371-R381 (2010).

19. Yoshihara, Y. Molecular genetic dissection of the zebrafish olfactory system. Results Probl. Cell. Differ. 47, 97-120 (2009).

20. Miyasaka, N. et al. From the olfactory bulb to higher brain centers: genetic visualization of secondary olfactory pathways in zebrafish. J. Neurosci. 29, 4756-4767 (2009).

21. Faedo, A. et al. Developmental expression of the T-box transcription factor T-bet/Tbx21 during mouse embryogenesis. Mech. Dev. 116, 157-160 (2002).

22. Mitsui, S., Igarashi, K. M., Mori, K. \& Yoshihara, Y. Genetic visualization of the secondary olfactory pathway in Tbx21 transgenic mice. Neural Syst Circuits 1, 5 (2011).

23. Braubach, O. R. et al. Experience-dependent versus experience-independent postembryonic development of distinct groups of zebrafish olfactory glomeruli. J. Neurosci. 33, 6905-6916 (2013).

24. Baier, H. \& Korsching, S. Olfactory glomeruli in the zebrafish form an invariant pattern and are identifiable across animals. J. Neurosci. 14, 219-230 (1994).

25. Braubach, O. R., Fine, A. \& Croll, R. P. Distribution and functional organization of glomeruli in the olfactory bulbs of zebrafish (Danio rerio). J. Comp. Neurol. 520, 2317-2339 (2012).

26. Concha, M. L. \& Wilson, S. W. Asymmetry in the epithalamus of vertebrates. J. Anat. 199, 63-84 (2001).

27. Concha, M. L. et al. Local tissue interactions across the dorsal midline of the forebrain establish CNS laterality. Neuron 39, 423-438 (2003).

28. Gamse, J. T., Thisse, C., Thisse, B. \& Halpern, M. E. The parapineal mediates leftright asymmetry in the zebrafish diencephalon. Development 130, 1059-1068 (2003).

29. Klein, S., Staring, M., Murphy, K., Viergever, M. A. \& Pluim, J. P. elastix: a toolbox for intensity-based medical image registration. IEEE. Trans. Med. Imaging. 29, 196-205 (2010).

30. Ward, Jr J. H. Hierarchical grouping to optimize an objective function. J. Am. Stat. Assoc. 58, 236-244 (1963).

31. Sato, Y., Miyasaka, N. \& Yoshihara, Y. Mutually exclusive glomerular innervation by two distinct types of olfactory sensory neurons revealed in transgenic zebrafish. J. Neurosci. 25, 4889-4897 (2005).

32. Koide, T. et al. Olfactory neural circuitry for attraction to amino acids revealed by transposon-mediated gene trap approach in zebrafish. Proc. Natl Acad. Sci. USA 106, 9884-9889 (2009).

33. Oka, Y., Saraiva, L. R. \& Korsching, S. I. Crypt neurons express a single V1R-related ora gene. Chem. Senses 37, 219-227 (2012).

34. Gayoso, J., Castro, A., Anadon, R. \& Manso, M. J. Crypt cells of the zebrafish Danio rerio mainly project to the dorsomedial glomerular field of the olfactory bulb. Chem. Senses 37, 357-369 (2012).

35. Ahuja, G. et al. Zebrafish crypt neurons project to a single, identified mediodorsal glomerulus. Sci. Rep. 3, 2063 (2013).

36. deCarvalho, T. N., Akitake, C. M., Thisse, C., Thisse, B. \& Halpern, M. E. Aversive cues fail to activate fos expression in the asymmetric olfactoryhabenula pathway of zebrafish. Front Neural Circuits 7, 98 (2013).

37. Tay, T. L., Ronneberger, O., Ryu, S., Nitschke, R. \& Driever, W. Comprehensive catecholaminergic projectome analysis reveals single-neuron integration of zebrafish ascending and descending dopaminergic systems. Nat. Commun. 2, 171 (2011)

38. Schweitzer, J., Lohr, H., Filippi, A. \& Driever, W. Dopaminergic and noradrenergic circuit development in zebrafish. Dev. Neurobiol. 72, 256-268 (2012).

39. Yaksi, E., von Saint Paul, F., Niessing, J., Bundschuh, S. T. \& Friedrich, R. W Transformation of odor representations in target areas of the olfactory bulb. Nat. Neurosci. 12, 474-482 (2009).

40. Wullimann, M. F. \& Mueller, T. Teleostean and mammalian forebrains contrasted: Evidence from genes to behavior. J. Comp. Neurol. 475, 143-162 (2004).

41. Nikonov, A. A., Finger, T. E. \& Caprio, J. Beyond the olfactory bulb: an odotopic map in the forebrain. Proc. Natl Acad. Sci. USA 102, 18688-18693 (2005).

42. Bianco, I. H. \& Wilson, S. W. The habenular nuclei: a conserved asymmetric relay station in the vertebrate brain. Philos. Trans. R. Soc. Lond. B. Biol. Sci. 364, 1005-1020 (2009).

43. Amo, R. et al. Identification of the zebrafish ventral habenula as a homolog of the mammalian lateral habenula. J. Neurosci. 30, 1566-1574 (2010).

44. Aizawa, H. et al. Laterotopic representation of left-right information onto the dorso-ventral axis of a zebrafish midbrain target nucleus. Curr. Biol. 15 238-243 (2005). 
45. Agetsuma, M. et al. The habenula is crucial for experience-dependent modification of fear responses in zebrafish. Nat. Neurosci. 13, 1354-1356 (2010).

46. Lee, A. et al. The habenula prevents helpless behavior in larval zebrafish. Curr. Biol. 20, 2211-2216 (2010).

47. Derjean, D. et al. A novel neural substrate for the transformation of olfactory inputs into motor output. PLoS Biol. 8, e1000567 (2010).

48. Lambert, A. M., Bonkowsky, J. L. \& Masino, M. A. The conserved dopaminergic diencephalospinal tract mediates vertebrate locomotor development in zebrafish larvae. J. Neurosci. 32, 13488-13500 (2012).

49. Caron, S. J., Ruta, V., Abbott, L. F. \& Axel, R. Random convergence of olfactory inputs in the Drosophila mushroom body. Nature 497, 113-117 (2013).

50. Gaudry, Q., Hong, E. J., Kain, J., de Bivort, B. L. \& Wilson, R. I. Asymmetric neurotransmitter release enables rapid odour lateralization in Drosophila. Nature 493, 424-428 (2013).

51. Kikuta, S. et al. Neurons in the anterior olfactory nucleus pars externa detect right or left localization of odor sources. Proc. Natl Acad. Sci. USA 107, 12363-12368 (2010).

52. Asakawa, K. et al. Genetic dissection of neural circuits by Tol 2 transposonmediated Gal4 gene and enhancer trapping in zebrafish. Proc. Natl Acad Sci. USA 105, 1255-1260 (2008).

53. Mattes, D., Haynor, D. R., Vesselle, H., Lewellen, T. K. \& Eubank, W. PET-CT image registration in the chest using free-form deformations. IEEE. Trans. Med. Imaging. 22, 120-128 (2003).

54. Schindelin, J. et al. Fiji: an open-source platform for biological-image analysis. Nat. Methods 9, 676-682 (2012).

55. Longair, M. H., Baker, D. A. \& Armstrong, J. D. Simple Neurite Tracer: open source software for reconstruction, visualization and analysis of neuronal processes. Bioinformatics 27, 2453-2454 (2011).

56. Schmid, B., Schindelin, J., Cardona, A., Longair, M. \& Heisenberg, M. A high-level 3D visualization API for Java and ImageJ. BMC Bioinformatics 11, 274 (2010).

\section{Acknowledgements}

We thank Rainer Friedrich and Charles Yokoyama for critical reading of the manuscript, Koichi Kawakami for reagents and transgenic fish, Shankar Srinivas and Stephen J. Smith for reagents, Toshiyuki Shiraki, Hisaya Kakinuma and Hitoshi Okamoto for assistance in generation and maintenance of transgenic fish, Tetsuya Koide for help in identification of zebrafish $t b \times 21$ gene and members of the Yoshihara laboratory for fish care and discussion. This work was supported in part by Grants-in-Aid for Scientific Research (22500303 and 25430025 to N.M.; 20300117 to Y.Y.) and for Scientific Research on Innovative Areas 'Fluorescence Live Imaging' (23113520 and 25113724 to N.M.), 'Systems Molecular Ethology' (23115723 to Y.Y.) and 'Memory Dynamism' (25115005 to Y.Y.) from the Ministry of Education, Culture, Sports, Science and Technology of Japan and a Grant from the Human Frontier Science Program (HFSP RGP0015/2010 to H.S.S and Y.Y.).

\section{Author contributions}

N.M. and Y.Y designed the study and wrote the manuscript. N.M., N.W. and M.M. performed the experiments. N.M., I.A.-C. and U.S. analysed the data. H.S.S. supervized the analysis and gave conceptual advice.

\section{Additional information}

Supplementary Information accompanies this paper at http://www.nature.com/ naturecommunications

Competing financial interests: The authors declare no competing financial interest.

Reprints and permission information is available online at http://npg.nature.com/ reprintsandpermissions/

How to cite this article: Miyasaka, N. et al. Olfactory projectome in the zebrafish forebrain revealed by genetic single-neuron labeling. Nat. Commun. 5:3639 doi: 10.1038/ncomms4639 (2014). 